\title{
Resolution of Nonsingularities for Mumford Curves
}

\author{
by
}

\author{
Emmanuel LEPAGE
}

\begin{abstract}
Given a Mumford curve $X$ over $\overline{\mathbf{Q}}_{p}$, we show that for every semistable model $\mathcal{X}$ of $X$ and every closed point $x$ of this semistable model, there exists a finite étale cover $Y$ of $X$ such that every semistable model of $Y$ over $\mathcal{X}$ has a vertical component above $x$. We then give applications of this to the tempered fundamental group. In particular, we prove that two punctured Tate curves $\overline{\mathbf{Q}}_{p}$ with isomorphic tempered fundamental groups are isomorphic over $\mathbf{Q}_{p}$.
\end{abstract}

2010 Mathematics Subject Classification: Primary 14G22; Secondary 14H30.

Keywords: tempered fundamental group, anabelian geometry, Berkovich geometry.

\section{Introduction}

In this article, given a hyperbolic curve $X$ over $\overline{\mathbf{Q}}_{p}$, we are interested in finding a finite étale cover $Y$ of this curve such that the stable reduction of the cover has irreducible components lying over the smooth locus of the stable reduction of $X$. Such techniques of resolution of nonsingularities are often used in anabelian geometry. We will apply our results to an anabelian study of the tempered fundamental group.

In [13, Th. 0.2], A. Tamagawa proved that for every hyperbolic curve $X=$ $\bar{X} \backslash D$ and every closed point $x$ of the stable reduction of $X$, there exists a finite étale cover $Y$ and an irreducible component $y$ of the stable reduction of $Y$ lying above $x$. We would like to generalize this to all the semistable reductions of $X$ : given a semistable model $\mathcal{X}$ of $X$ and a closed point $x$ of the special fiber $\mathcal{X}_{s}$ of $X$, is there a finite étale cover $Y$ and an irreducible component $y$ of the minimal semistable model $\mathcal{Y}$ of $Y$ above $\mathcal{X}$ such that $y$ lies above $x$ ? To give an example of anabelian motivation for this kind of resolution of nonsingularities, as shown by F. Pop and

Communicated by A. Tamagawa. Received November 30, 2011. Revised September 7, 2012.

E. Lepage: Université Pierre et Marie Curie, 4 place Jussieu, 75005 Paris, France;

e-mail: lepage@math.jussieu.fr

(C) 2013 Research Institute for Mathematical Sciences, Kyoto University. All rights reserved. 
J. Stix in [12, Cor. 41], if $X_{0}$ is a geometrically connected hyperbolic curve over a finite extension $K$ of $\mathbf{Q}_{p}$ such that $X_{0, \overline{\mathbf{Q}}_{p}}$ satisfies this kind of resolution of nonsingularities, then every section of $\pi_{1}^{\mathrm{alg}}\left(X_{0}\right) \rightarrow \operatorname{Gal}\left(\overline{\mathbf{Q}}_{p} / K\right)$ has its image in a decomposition group of a unique valuative point. In this article, we will prove that this resolution of nonsingularities is satisfied by Mumford curves.

First, let us translate this into an analytic setting. Let $X^{\text {an }}$ be the Berkovich space of $X$. Given a semistable model $\mathcal{X}$ of $X$, there is a reduction map $\pi_{\mathcal{X}}$ : $X^{\text {an }} \rightarrow \mathcal{X}_{s}$. If $\eta_{y}$ is the generic point of an irreducible component of $\mathcal{Y}$, the subset $\pi_{\mathcal{Y}}^{-1}\left(\eta_{y}\right) \subset Y^{\text {an }}$ reduces to a single point. We denote by $V(\mathcal{Y})$ the set of points of $Y^{\text {an }}$ whose image under $\pi_{\mathcal{Y}}$ is a generic point. Therefore our question reduces to the following: is there a $Y$ and an element of $V(\mathcal{Y})$ which is mapped to $\pi_{\mathcal{X}}^{-1}(x)$ ? Since $V(\mathcal{Y})$ contains $V(Y):=V\left(\mathcal{Y}_{0}\right)$ where $\mathcal{Y}_{0}$ is the stable model of $Y$ and $\pi_{\mathcal{X}}^{-1}(x)$ is a nonempty open subset of $X^{\text {an }}$, it is enough to show that the union $\widetilde{V}(X)$ of the images of $V(Y)$ in $X^{\text {an }}$, where $Y$ runs over all finite étale covers of $X$, is dense in $X^{\text {an }}$.

Theorem 0.1 (Th. 2.7). Let $X$ be a hyperbolic Mumford curve. Then $\widetilde{V}(X)$ is dense in $X^{\text {an }}$.

As a consequence, by considering the étale locus of a map to $\mathbf{P}^{1}$, one finds that any curve over $\overline{\mathbf{Q}}_{p}$ has a dense open subset which satisfies this resolution of nonsingularities.

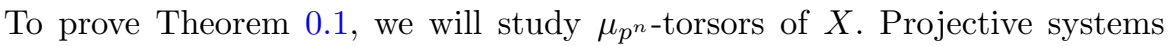
of $\mu_{p^{n}}$-torsors of $X$ are classified by $H^{1}\left(X, \mathbf{Z}_{p}(1)\right)$. Let $c$ be an element of $H^{1}\left(X, \mathbf{Z}_{p}(1)\right)$. Let $x$ be a $\mathbf{C}_{p}$-point of $X$. There is a small rigid neighborhood $D$ of $x$ in $X_{\mathbf{C}_{p}}$ isomorphic to a disk and a morphism $f: D \rightarrow \mathbf{G}_{\mathrm{m}}$, i.e. an element $f \in O^{*}(D)$, such that the restriction of $c$ to $D$ is the pullback of the canonical element of $H^{1}\left(\mathbf{G}_{\mathrm{m}}, \mathbf{Z}_{p}(1)\right)$. Let $Y_{n} \rightarrow X$ be the $\mu_{p^{n}}$-torsor induced by $c$. For $n$ large enough, there is a smallest closed disk $D_{n}$ of $D$ centered at $x$ such that $Y_{n \mid D_{n}} \rightarrow X_{n \mid D_{n}}$ is a nontrivial cover. Then the behavior of the restriction of $Y_{n}$ to the Berkovich generic point $x_{n}$ of $D_{n}$ for $n$ large enough only depends on the ramification index $e$ of $f: D \rightarrow \mathbf{G}_{\mathrm{m}}$ at $x$. More precisely, if $y_{n}$ is a preimage of $x_{n}$ in $Y_{n}$, the extension $\mathcal{H}\left(y_{n}\right) / \mathcal{H}\left(x_{n}\right)$ of complete residue fields induces an extension $k\left(y_{n}\right) / k\left(x_{n}\right)$ of their reductions in characteristic $p$. This extension is an Artin-Schreier extension: $k\left(x_{n}\right)$ is isomorphic to $\overline{\mathbf{F}}_{p}(X)$ and $k\left(y_{n}\right)$ is isomorphic to $k\left(x_{n}\right)[Y] /\left(Y^{p}-Y-X^{e}\right)$. The general study of Artin-Schreier extensions tells us that if $e$ is not a power of $p$, then $k\left(y_{n}\right)$ is not a rational extension of $\overline{\mathbf{F}}_{p}$. This implies that $y_{n} \subset V\left(Y_{n}\right)$ and $x_{n} \in \widetilde{V}(X)$, and therefore $x$ lies in the closure of $\widetilde{V}(X)$. 
Hodge-Tate theory yields a canonical decomposition $H^{1}\left(X, \mathbf{Z}_{p}(1)\right) \otimes \mathbf{z}_{p} \mathbf{C}_{p}$ $=H^{1}\left(X_{\mathbf{C}_{p}}, O_{X_{\mathbf{C}_{p}}}\right)(1) \oplus H^{0}\left(X_{\mathbf{C}_{p}}, \Omega_{X_{\mathbf{C}_{p}}}\right)$. Let us consider the induced map $p$ : $H^{1}\left(X, \mathbf{Z}_{p}(1)\right) \rightarrow H^{0}\left(X_{\mathbf{C}_{p}}, \Omega_{X_{\mathbf{C}_{p}}}\right)$. If $c \in H^{1}\left(X, \mathbf{Z}_{p}(1)\right)$ The restriction of $p(c)$ to $D$ is $\frac{d f}{f}$. When $X$ is a Mumford curve over $\overline{\mathbf{Q}}_{p}$, the image of $p$ lies in $H^{0}\left(X, \Omega_{X}\right)$. Let $\Omega \subset \mathbf{P}^{1}$ be the universal topological cover of $X$. If $x \in \Omega\left(\overline{\mathbf{Q}}_{p}\right)$, one can find a rational function $f$ with neither poles nor zeroes in $\Omega$ such that $\frac{d f}{f}$ has a zero at $x$ with multiplicity $m$ such that $m+1$ is not a power of $p$. Let $c_{f}$ be the pullback of the canonical element of $H^{1}\left(\mathbf{G}_{\mathrm{m}}, \mathbf{Z}_{p}(1)\right)$ along $f: \Omega \rightarrow \mathbf{G}_{\mathrm{m}}$, and let $x_{n} \in \Omega$ be defined as previously. Then for the topology of uniform convergence on every compact subset of $O^{*}(D)$, we will approximate $f$ by elements of $\bigcup_{X^{\prime}} \Theta\left(X^{\prime}\right)$, where $X^{\prime}$ runs over finite topological pointed covers of $X$, and $\Theta\left(X^{\prime}\right)$ is the set of theta functions of $X^{\prime}$. Using this, one can construct for every $n$ a finite topological cover $X^{\prime}$ of $X$ and a $\mu_{p^{n}}$-torsor $Y \rightarrow X^{\prime}$ such that the preimage in $Y$ of the image in $X^{\prime}$ of $x_{n}$ lies in $V(Y)$. Therefore $x \in \widetilde{V}(X)$. Since $X_{\overline{\mathbf{Q}}_{p}}$ is dense in $X^{\text {an }}$, one gets the density of $\widetilde{V}(X)$.

In a second part, we use the resolution of nonsingularities to study the tempered fundamental group. We show the following:

Theorem 0.2 (Th. 3.10). Let $X_{1}$ and $X_{2}$ be two Mumford curves over $\overline{\mathbf{Q}}_{p}$. Given an isomorphism between their tempered fundamental groups, there is a canonical homeomorphism between their Berkovich spaces.

The strategy is the following. For every semistable model of a curve $X$, there is a retraction from $\bar{X}^{\text {an }}$ to the graph of this semistable reduction. One gets a map from $\bar{X}^{\text {an }}$ to the projective limit of the graphs of the semistable reductions, which is a homeomorphism. If $X$ is a Mumford curve, by resolution of nonsingularities, semistable models of the form $\mathcal{Y} / G$, where $Y$ runs over finite Galois cover of $X, \mathcal{Y}$ is the stable model of $Y$ and $G=\operatorname{Gal}(Y / X)$, are cofinal among semistable models of $X$. However, a theorem of S. Mochizuki tells us that one can recover the graph of the stable reduction from the tempered fundamental group ([11, Cor. 3.11]). Therefore if $Y_{1}$ is a finite Galois cover of $X_{1}$, and $Y_{2}$ is the corresponding finite Galois cover given by the isomorphism of tempered fundamental groups, then the graph $\mathbb{G}_{Y_{1}}$ of $\mathcal{Y}_{1}$ is canonically isomorphic to the graph $\mathbb{G}_{Y_{2}}$ of $\mathcal{Y}_{2}$, and one gets a similar isomorphism after quotienting by $\operatorname{Gal}\left(Y_{1} / X_{1}\right) \simeq \operatorname{Gal}\left(Y_{2} / X_{2}\right)$. The problem is to recover from the tempered fundamental group the transition maps between the geometric realisation of the different graphs.

At the end of the article, we will be interested in the anabelianness of the tempered fundamental group for punctured Tate curves: 
Theorem 0.3 (Th. 4.1). Let $q_{1}, q_{2} \in \overline{\mathbf{Q}}_{p}$ be such that $\left|q_{1}\right|,\left|q_{2}\right|<1$. Assume there is an isomorphism $\psi$ between the tempered fundamental groups of $\left(\mathbf{G}_{\mathrm{m}} / q_{1}^{\mathbf{Z}}\right) \backslash\{1\}$ and $\left(\mathbf{G}_{\mathrm{m}} / q_{2}^{\mathbf{Z}}\right) \backslash\{1\}$. Then there exists $\sigma \in \operatorname{Gal}\left(\overline{\mathbf{Q}}_{p} / \mathbf{Q}_{p}\right)$ such that $q_{2}=\sigma\left(q_{1}\right)$.

However, the proof of this result does not give any particular element of $\operatorname{Gal}\left(\overline{\mathbf{Q}}_{p} / \mathbf{Q}_{p}\right)$.

Let $\Omega_{i}=\mathbf{G}_{\mathrm{m}} \backslash\left\{q_{i}^{n}\right\}_{n \in \mathbf{Z}}$ and $X_{i}=\Omega_{i} / q_{i}^{\mathbf{Z}}$. According to Theorem 0.2, the isomorphism of tempered fundamental groups induces a homeomorphism $\bar{\psi}$ : $\mathbf{G}_{\mathrm{m}}^{\mathrm{an}} \rightarrow \mathbf{G}_{\mathrm{m}}^{\mathrm{an}}$ which maps $q_{1}^{n}$ to $q_{2}^{n}$ for every $n \in \mathbf{Z}$.

Elements of $O^{*}\left(\Omega_{i}\right)$ correspond, up to a scalar, to a current on the semitree $\mathbb{T}_{i}$ of $\Omega_{i}$. Since $\psi$ induces an isomorphism $\mathbb{T}_{1} \simeq \mathbb{T}_{2}$, one gets a group isomorphism $\alpha: O^{*}\left(\Omega_{1}\right) \rightarrow O^{*}\left(\Omega_{2}\right)$. The crucial point will be to show that for every $f \in O^{*}\left(\Omega_{1}\right)$ and $z \in \mathbf{G}_{\mathrm{m}}(\mathbf{C})$, the multiplicity of $\frac{d f}{f}$ at $z$ coincides with the multiplicity of $\frac{d \alpha(f)}{\alpha(f)}$ at $\bar{\psi}(z)$. By density of $\mathbf{Z}$ in $\mathbf{Z}_{p}$, one also gets a similar result for $\mathbf{Z}_{p}$-linear combinations of differential 1-forms like $\frac{d f}{f}$. Once one knows this, one can build, for every $n \in \mathbf{N}$, an element $f \in O^{*}\left(\Omega_{1}\right)$ such that $\frac{d f}{f}(1)=q_{1}^{n}$ and $\frac{d \alpha(f)}{\alpha(f)}(1)=q_{2}^{n}$ : one then deduces that for every $P \in \mathbf{Z}_{p}[X], P\left(q_{1}\right)=0$ if and only if $P\left(q_{2}\right)=0$.

\section{$\S 1$. Berkovich geometry of curves}

In the following, $K=\mathbf{C}_{p}$, and $k$ is its residue field, which is isomorphic to $\overline{\mathbf{F}}_{p}$. The norm will be chosen so that $|p|=p^{-1}$, and the valuation so that $v(p)=1$. All valued fields will have valuations with values in $\mathbf{R}_{\geq 0}$.

If $X$ is an algebraic variety over $K$, one can associate to $X$ a topological set $X^{\text {an }}$ with a continuous map $\phi: X^{\text {an }} \rightarrow X$ defined in the following way. A point of $X^{\text {an }}$ is an equivalence class of morphisms $\operatorname{Spec} K^{\prime} \rightarrow X$ over $\operatorname{Spec} K$ where $K^{\prime}$ is a complete valued extension of $K$. Two morphisms Spec $K^{\prime} \rightarrow X$ and Spec $K^{\prime \prime} \rightarrow X$ are equivalent if there exists a common valued extension $L$ of $K^{\prime}$ and $K^{\prime \prime}$ such that

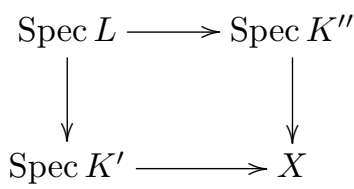

commutes. In fact, for any $x \in X^{\text {an }}$, there is a unique smallest such complete valued field defining $x$, denoted by $\mathcal{H}(x)$ and called the completed residue field of $x$. We denote by $k(x)$ the residue field of $\mathcal{H}(x)$ and by $\operatorname{val}(x) \subset \mathbf{R}_{>0}$ the group of values of $\mathcal{H}(x)$. Forgetting the valuation, one gets points $\operatorname{Spec}(K) \rightarrow X$ from the same equivalence class of points; this defines a point of $X$, hence a map $X^{\text {an }} \rightarrow X$. If $U=\operatorname{Spec} A$ is an affine open subset of $X$, then every $x \in \phi^{-1}(U)$ defines a 
seminorm $\mid{ }_{x}$ on $A$. The topology on $\phi^{-1}(U)$ is defined to be the coarsest such that $x \mapsto|f|_{x}$ is continuous for every $f \in A$.

The space $X^{\text {an }}$ is locally compact, and even compact if $X$ is proper. In fact $X^{\text {an }}$ is more than just a topological space: it can be enriched into a $K$-analytic space, as defined by Berkovich in [2].

Points of $\mathbf{A}^{1, \text { an }}$ are of four different types and are described in the following way:

- A closed ball $B=B(a, r) \subset \mathbf{C}_{p}$ of center $a$ and radius $r$ defines a point $b=b_{a, r}$ of $\mathbf{A}^{1, \text { an }}$, called the Gauss point of $B$, by

$$
|f|_{b}=\sup _{x \in B}|f(x)|=\max _{i \in \mathbf{N}}\left|a_{i}\right| r^{i} \quad \text { if } f=\sum_{i \in \mathbf{N}} a_{i} X^{i} .
$$

The point $b_{a, r}$ is said to be of type 1 if $r=0$, of type 2 if $r \in p^{\mathbf{Q}}$, and of type 3 otherwise. The pairs $(a, r)$ and $\left(a^{\prime}, r^{\prime}\right)$ define the same point if and only if $B(a, r)=B\left(a^{\prime}, r^{\prime}\right)$, i.e. $r=r^{\prime}$ and $\left|a-a^{\prime}\right| \leq r$.

- A decreasing family $E=\left(B_{i}\right)_{i \in I}$ of balls with empty intersection defines a point by

$$
|f|_{E}=\inf _{i \in I}|f|_{b_{i}}
$$

where $b_{i}$ is the Gauss point of $B_{i}$. Such a point is said to be of type 4 .

If $r \in p^{\mathbf{Q}}$ and $a \in \mathbf{C}_{p}$ are such that $|a|=r$, then $|X / a|_{b_{a, r}}=1$ and $k\left(b_{a, r}\right)=$ $k(\overline{X / a})$.

The classification by type of points can be generalized to curves in such a way that it is preserved by finite morphisms. A point $x$ is:

- of type 1 if $\mathcal{H}(x)=\mathbf{C}_{p}$;

- of type 2 if $\operatorname{deg} \operatorname{tr} k(x) / \overline{\mathbf{F}}_{p}=1$;

- of type 3 if $\operatorname{val}(x) \neq p^{\mathbf{Q}}$;

- of type 4 otherwise (i.e. $\mathcal{H}(x) / \mathbf{C}_{p}$ is an immediate extension).

If $x$ is of type 2, we denote by $g_{k(x)}$ the genus of the proper $\overline{\mathbf{F}}_{p}$-curve whose field of fractions is $k(x)$.

Let $X$ be a proper and smooth $K$-curve. The topological space $X$ is a quasipolyhedron in the sense of [2, Def. 4.1.1]: there exists a base of open subsets $U$ such that:

- $\bar{U} \backslash U$ is finite;

- $U$ is countable at infinity;

- for every $x \neq y \in U$, there exists a unique subset $[x, y] \subset U$ that is homeomorphic to $[0,1]$ with endpoints $x$ and $y$. 
A quasipolyhedron that satisfies these three properties is said to be simply connected.

If $X$ is a curve, the set of points of type different from 2 is totally disconnected. Therefore, on every nonconstant path, there exist infinitely many points of type 2 .

The topological universal cover $X^{\infty}$ is a simply connected quasipolyhedron. Therefore any subset $I$ of $X^{\infty}$ is contained in a smallest connected subset Conv $(I)$ $=\bigcup_{(x, y) \in I^{2}}[x, y]$. If $I, J$ are closed connected subsets of $X^{\infty}$, we denote $[I, J]=$ $\bigcap_{(x, y) \in I \times J}[x, y]$. This set is homeomorphic to $[0,1]$ if $I \cap J=\emptyset$ and $I \cap[I, J]$ and $J \cap[I, J]$ each reduce to a point. If $x \in X^{\infty}$ and $I$ is a closed subset of $X^{\infty}$, we denote by $r_{I}(x)$ the unique element of $[x, I] \cap I$. The map $r_{I}: X^{\infty} \rightarrow I$ is a continuous retraction of the embedding $I \rightarrow X^{\infty}$.

Let $\mathcal{X}$ be a semistable model. There is a specialization map $\pi_{\mathcal{X}}: X^{\text {an }} \rightarrow \mathcal{X}_{k}$ defined in the following way. If $x \in X^{\text {an }}$, the morphism Spec $\mathcal{H}(x) \rightarrow X$ extends to a morphism $\operatorname{Spec} O_{\mathcal{H}(x)} \rightarrow \mathcal{X}$ by properness of $\mathcal{X} \rightarrow \operatorname{Spec} O_{K}$, hence to a morphism Spec $k(x) \rightarrow \mathcal{X}_{k}$; the image of this morphism is $\pi_{\mathcal{X}}(x)$. This specialization map is anticontinuous: the preimage of a closed subset is an open subset.

If $z$ is the generic point of an irreducible component of $\mathcal{X}_{k}$, then $z$ is of codimension 1 in $\mathcal{X}$ and thus $O_{\mathcal{X}, z}$ is a valuation ring. The completion of Frac $O_{\mathcal{X}, z}$ defines a point $b_{z}$ of $X^{\text {an }}$ which is the unique element of $\pi_{\mathcal{X}}^{-1}(z)$. We denote by $V(\mathcal{X}) \subset X^{\text {an }}$ the set of such $b_{z}$, and by $V(\mathcal{X})^{\infty}$ its preimage in $X^{\infty}$.

One has $X^{\text {an }} \backslash V(\mathcal{X})=\coprod \pi_{\mathcal{X}}^{-1}(x)$ where $x$ runs through closed points of $\mathcal{X}_{k}$, and $\pi_{\mathcal{X}}^{-1}(x)$ is open by anticontinuity of $\pi_{\mathcal{X}}$. In particular, if $z, z^{\prime} \in X^{\text {an }}$ are such that $\pi_{\mathcal{X}}(z) \neq \pi_{\mathcal{X}}\left(z^{\prime}\right)$, then every path joining $z$ to $z^{\prime}$ meets $V(\mathcal{X})$.

We denote $S(\mathcal{X})^{\infty}=\operatorname{Conv}\left(V(\mathcal{X})^{\infty}\right)$ and let $r_{\mathcal{X}}^{\infty}$ be the retraction $r_{\mathcal{X}}^{\infty}: X^{\infty} \rightarrow$ $S(\mathcal{X})^{\infty}$ of the embedding $\iota_{\mathcal{X}}^{\infty}: S(\mathcal{X})^{\infty} \rightarrow X^{\infty}$. Since $V(\mathcal{X})^{\infty}$ is $\operatorname{Gal}\left(X^{\infty} / X\right)$ invariant, $S(\mathcal{X})^{\infty}$ is also $\operatorname{Gal}\left(X^{\infty} / X\right)$-invariant and $r_{\mathcal{X}}^{\infty}$ is $\operatorname{Gal}\left(X^{\infty} / X\right)$-equivariant. We denote by $S(\mathcal{X})$ the image of $S(\mathcal{X})^{\infty}$ in $X^{\text {an }}$; it is called the skeleton of $\mathcal{X}$. We denote by $r_{\mathcal{X}}$ the retraction $X^{\text {an }} \rightarrow S(\mathcal{X})$ induced by $r_{\mathcal{X}}^{\infty}$. The space $S(\mathcal{X})$ is compact and the inclusion map $\iota_{\mathcal{X}}: S(\mathcal{X}) \rightarrow X^{\text {an }}$ is a homotopy equivalence. In fact $S(\mathcal{X})$ is characterized by the fact that it is the smallest subset $S$ of $X^{\text {an }}$ that contains $V(\mathcal{X})$ and is such that $S \rightarrow X^{\text {an }}$ is a homotopy equivalence.

If $z$ is a node of $\mathcal{X}_{k}$, then $\pi^{-1}(z)$ is an open annulus. It contains a unique closed connected subset $S_{z}$ homeomorphic to $\mathbf{R}$. More precisely, if one chooses an isomorphism of analytic spaces $\pi^{-1}(z) \simeq\left\{\left.z \in \mathbf{A}^{1 \text {,an }}\left|r_{0}<\right| T\right|_{z}<1\right\}$, then $S_{z}=\left\{b_{0, r} \mid r_{0}<r<1\right\}$ (in particular the points of type 2 of $S_{z}$ can be identified with $\left.\mathbf{Q} \cap\left(r_{0}, 1\right)\right)$. If $z$ is a closed point of the smooth locus of $\mathcal{X}_{k}$, then $\pi^{-1}(z)$ is an open disk. Since, for every point $b$ of type 2 of disks and annuli, $k(b)$ is a rational 
extension of $k$, one finds that if $x \in X^{\text {an }}$ is a point of type 2 such that $g_{k(x)} \neq 0$, then $x \in V(\mathcal{X})$.

One recovers $S(\mathcal{X})$ as the union of $V(\mathcal{X})$ and of $S_{z}$ for every node $z$ of $\mathcal{X}_{k}$. One deduces that $S(\mathcal{X})$ is homeomorphic to the dual graph $\mathbb{G}_{\mathcal{X}}$ of $\mathcal{X}_{k}$. Similarly $S(\mathcal{X})^{\infty}$ is homeomorphic to the universal cover $\mathbb{T}_{\mathcal{X}}$ of $\mathbb{G}_{\mathcal{X}}$.

If $X_{1} \rightarrow X_{2}$ is a morphism of proper and smooth $K$-curves and $\mathcal{X}_{1} \rightarrow \mathcal{X}_{2}$ is an extension to semistable $O_{K}$-models, then

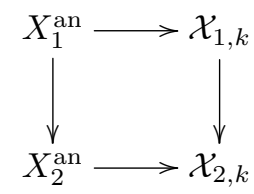

is commutative.

Assume now that $\mathcal{X}_{1} \rightarrow \mathcal{X}_{2}$ is a morphism of semistable models of the same curve $X$. If $z_{1}$ is the generic point of an irreducible component of $\mathcal{X}_{1, k}$ which maps to the generic point $z_{2}$ of an irreducible component of $\mathcal{X}_{2, k}$, the previous diagram tells us that $b_{z_{1}}=b_{z_{2}}$. Since $\mathcal{X}_{1} \rightarrow \mathcal{X}_{2}$ is surjective, one gets $V\left(\mathcal{X}_{2}\right) \subset V\left(\mathcal{X}_{1}\right) \subset$ $S\left(\mathcal{X}_{1}\right)$. Since $S\left(\mathcal{X}_{2}\right)$ is the smallest subset of $X^{\text {an }}$ that contains $V\left(\mathcal{X}_{2}\right)$ and is such that $S\left(\mathcal{X}_{2}\right) \rightarrow X^{\text {an }}$ is a homotopy equivalence, one finds that $S\left(\mathcal{X}_{2}\right) \subset S\left(\mathcal{X}_{1}\right)$. Similarly, $V\left(\mathcal{X}_{2}\right)^{\infty} \subset V\left(\mathcal{X}_{1}\right)^{\infty}$ and $S\left(\mathcal{X}_{2}\right)^{\infty} \subset S\left(\mathcal{X}_{1}\right)^{\infty}$.

Therefore, for every $x \in X^{\text {an }},\left[x, S\left(\mathcal{X}_{1}\right)\right] \subset\left[x, S\left(\mathcal{X}_{2}\right)\right]$, and thus $\iota \mathcal{X}_{1} r_{\mathcal{X}_{1}}(x) \in$ $\left[x, S\left(\mathcal{X}_{2}\right)\right]$. This implies that $r_{\mathcal{X}_{2}} \iota \mathcal{X}_{1} r_{\mathcal{X}_{1}}=r_{\mathcal{X}_{2}}$. Therefore, the maps $r_{\mathcal{X}_{1} / \mathcal{X}_{2}}:=$ $r_{\mathcal{X}_{2}} \iota \mathcal{X}_{1}: S\left(\mathcal{X}_{1}\right) \rightarrow S\left(\mathcal{X}_{2}\right)$ are compatible with composition and $(S(\mathcal{X}))_{\mathcal{X}}$ is a projective system of topological spaces. The maps $\left(r_{\mathcal{X}}\right)$ induce a continuous map

$$
r_{X}: X^{\text {an }} \rightarrow \lim _{\mathcal{X}} S(\mathcal{X})
$$

Proposition 1.1. The map $r_{X}$ is a homeomorphism.

Proof. Since $X^{\text {an }}$ is compact, the surjectivity of $r_{X}$ follows from the surjectivity of each $r_{\mathcal{X}}$.

Let $x \neq x^{\prime}$ be such that $r_{\mathcal{X}_{0}}(x)=r_{\mathcal{X}_{0}}\left(x^{\prime}\right)$ where $\mathcal{X}_{0}$ is the minimal model of $X$. Let $U$ be a simply connected open neighborhood of $r_{\mathcal{X}_{0}}(x)$ in $S\left(\mathcal{X}_{0}\right)$, and $V=r_{\mathcal{X}_{0}}^{-1}(U)$. Since $V$ is a simply connected quasipolyhedron, there is a minimal connected subset $\left[x, x^{\prime}\right] \subset V$ containing $x$ and $x^{\prime}$. It is homeomorphic to $[0,1]$ and has a natural order that makes $x$ the smallest element. Let $x_{1}<x_{2} \in\left[x, x^{\prime}\right]$ be points of type 2 .

Since $x_{i}$ is of type $2, V \backslash\left\{x_{i}\right\}$ has infinitely many components. Since $\overline{\mathbf{Q}}_{p}$-points are dense in $X^{\text {an }}$ one can find $z_{i, 1}, z_{i, 2}, z_{i, 3} \in V \cap X\left(\overline{\mathbf{Q}}_{p}\right)$ lying in different connected components of $X^{\text {an }} \backslash\left\{x_{i}\right\}$. Let $\mathcal{X}$ be the stable model of the marked curve 
$\left(X,\left\{z_{i j}\right\}_{i=1,2 ; j=1,2,3}\right.$. Since $\pi_{\mathcal{X}}\left(z_{i, 1}\right) \neq \pi_{\mathcal{X}}\left(z_{i, 2}\right)$, one has $S(\mathcal{X}) \cap\left[z_{i, 1}, z_{i, 2}\right] \neq \emptyset$ and therefore $r_{\mathcal{X}}\left(z_{i, 1}\right) \in\left[z_{i, 1}, z_{i, 2}\right]$.

Therefore, replacing $z_{i, 2}$ by $z_{y, 3}$, one finds that $r_{\mathcal{X}}\left(z_{i, 1}\right) \in\left[z_{i, 1}, z_{i, 2}\right] \cap\left[z_{i, 1}, z_{i, 3}\right]$ $=\left[z_{i, 1}, x_{i}\right]$ and similarly $r_{\mathcal{X}}\left(z_{i, 2}\right) \in\left[z_{i, 2}, x_{i}\right]$. Since $S(\mathcal{X})$ is connected and intersects $\left[z_{i, 1}, x_{i}\right]$ and $\left[z_{i, 2}, x_{i}\right]$, it follows that $x_{i} \in S(\mathcal{X})$. Therefore, in $\left[x, x^{\prime}\right]$, one has $r_{\mathcal{X}}(x) \leq x_{1}<x_{2} \geq r_{\mathcal{X}}\left(x^{\prime}\right)$, which proves the injectivity of $r_{X}$.

Since $X^{\text {an }}$ is compact and $\lim _{\mathcal{X}} S(\mathcal{X})$ is Hausdorff, $r_{X}$ is a homeomorphism.

Let $\mathcal{X}_{1} \rightarrow \mathcal{X}_{2}$ be a morphism of semistable models of $X$. Let $z$ be a node of $\mathcal{X}_{2, k}$. We will use the notation

$$
A_{z, \mathcal{X}_{1}}:=V\left(\mathcal{X}_{1}\right) \cap S_{z}
$$

If one chooses an orientation of $S_{z} \simeq \mathbf{R}$, then $A_{z, \mathcal{X}_{1}}$ becomes an ordered set. We denote $A_{z}=\bigcup_{\mathcal{X}_{1}} A_{z, \mathcal{X}_{1}} \subset S_{z}$.

\section{$\S 2$. Resolution of nonsingularities}

\section{$\S 2.1$. Definition}

If $X=\bar{X} \backslash D$ is a hyperbolic curve over $K$, we denote by $V(X)$ the subset $V(\overline{\mathcal{X}})$ of $\bar{X}^{\text {an }}$ (it is also a subset of $X^{\text {an }}$ since all of its points are of type 2 ), where $(\overline{\mathcal{X}}, \mathcal{D})$ is the stable model of $(\bar{X}, D)$, and we also denote by $\mathbb{G}_{X}$ the dual graph of $\overline{\mathcal{X}}_{k}$ and by $\mathbb{T}_{X}$ its universal cover.

Let $X=\bar{X} \backslash D$ be a hyperbolic curve over $K$. Let $X_{(2)}^{\text {an }} \subset X^{\text {an }}$ be the subset of type 2 points.

Let $\widetilde{V}(X)$ be the set of points $x$ of $X^{\text {an }}$ such that there exists a finite étale cover $f: Y \rightarrow X$ and $y \in V(Y)$ such that $f(y)=x$. If $x \in \tilde{V}(X)$, then $Y$ can be chosen to be Galois, so that in particular $f^{-1}(x) \subset V(Y)$, since $V(Y)$ is Galois equivariant. Let $\bar{V}(X)$ be the closure of $\widetilde{V}(X)$ in $\bar{X}$. If $f: Y \rightarrow X$ is a finite étale cover, then $\widetilde{V}(Y)=f^{-1}(\widetilde{V}(X))$ and $\bar{V}(Y)=f^{-1}(\bar{V}(X))$. One has $\widetilde{V}(X) \subset X_{(2)}^{\text {an }}$.

Definition 2.1. We say that $X$ satisfies resolution of nonsingularities if

$$
\widetilde{V}(X)=X_{(2)}^{\text {an }} .
$$

Proposition 2.2. Let $X=\bar{X} \backslash D$ be a curve. The following are equivalent:

(i) $\widetilde{V}(X)=X_{(2)}^{\text {an }}$;

(ii) $\bar{V}(X)=\bar{X}^{\mathrm{an}}$;

(iii) $X(K) \subset \bar{V}(X)$. 
Proof. (i) $\Rightarrow$ (ii). Points of type 2 are dense in $X_{\mathrm{an}}$.

(ii) $\Rightarrow$ (iii) is obvious.

(iii) $\Rightarrow(\mathrm{i})$. Let $x \in X_{(2)}$. Then $X^{\text {an }} \backslash\{x\}$ has infinitely many components and they are open in $X^{\text {an }}$. Since $X(K)$ is dense in $X^{\text {an }}$, each of these components intersects $\widetilde{V}(X)$. Let $x_{1}, x_{2}, x_{3} \in \widetilde{V}(X)$ lie in different connected components $S_{1}, S_{2}, S_{3}$ of $X^{\text {an }} \backslash\{x\}$. Let $f: Y \rightarrow X$ be a finite cover such that there is $y_{i}$ over $x_{i}$ lying in $S(Y)$ for $i=1,2,3$. Up to replacing $Y$ by a Galois closure, one can assume that $Y \rightarrow X$ is Galois. Since the image $T$ of $S(Y)$ in $X^{\text {an }}$ is connected and $x_{1} x_{2} \in T$, one has $x \in T$. Since $S(Y) \subset Y^{\text {an }}$ is $\operatorname{Gal}(Y / X)$-invariant, $S(Y)=f^{-1}(T)$. Let $y \in f^{-1}(x)$. For any neighborhood $U$ of $x, U \cap T \cap S_{i} \neq \emptyset$. Thus for any neighborhood $V$ of $y$ small enough (for example, such that $f^{-1}(x) \cap V=\{y\}$ ), the set $V \cap S(Y) \backslash\{y\}$ has at least three connected components. Thus $y \in V(Y)$ and $x \in \widetilde{V}(X)$.

If $X$ is a curve over $\overline{\mathbf{Q}}_{p}$, then $X\left(\overline{\mathbf{Q}}_{p}\right)$ is dense in $X\left(\overline{\mathbf{C}}_{p}\right)$, thus $X$ satisfies resolution of nonsingularities if and only if $X\left(\overline{\mathbf{Q}}_{p}\right) \subset \bar{V}(X)$.

If $Y \rightarrow X$ is a morphism of hyperbolic curves over $\overline{\mathbf{Q}}_{p}$, and $\mathcal{X}$ is a semistable model of $\bar{X}$, then there exists a minimal semistable model $\mathcal{Y}$ of $Y$ above $\mathcal{X}$ $(\mathcal{Y}$ is the stable marked hull of the normalization of $\mathcal{X}$ in $K(Y)$, in the sense of $[10$, Cor. 2.20]).

Proposition 2.3. Let $X$ be a $\overline{\mathbf{Q}}_{p}$-curve which satisfies resolution of nonsingularities. Let $\mathcal{X}$ be a semistable model of $\bar{X}$ and let $x$ be a closed point of $\mathcal{X}_{k}$. There exists a finite cover $Y \rightarrow X$ such that $\mathcal{Y}_{k}$ has a vertical component above $x$, where $\mathcal{Y}$ is the minimal semistable model of $Y$ above $\mathcal{X}$.

Proof. Let $\pi_{\mathcal{X}}: \bar{X}^{\text {an }} \rightarrow \mathcal{X}_{k}$ be the specialization map. Since $x$ is closed, $\pi_{\mathcal{X}}^{-1}(x)$ is open, and therefore contains a point $\tilde{x}$ of type 2 . Let $Y$ be a cover of $X$ and let $\tilde{y} \in V(Y)$ be above $\tilde{x}$. Then, for the stable model $\mathcal{Y}_{0}$ of $Y, \tilde{y}$ specializes via $\pi_{\mathcal{Y}_{0}}$ to a generic point of $\mathcal{Y}_{0, k}$. Therefore, $\tilde{y}$ specializes to a generic point for every semistable model of $Y$, in particular for $\mathcal{Y}$. Then $y:=\pi_{\mathcal{Y}}(\tilde{y})$ is mapped to $x$, and therefore the closure of $y$ is a vertical component above $x$.

\section{$\S 2.2$. Splitting points of torsors}

The map $\mathbf{G}_{\mathrm{m}} \stackrel{()^{n}}{\longrightarrow} \mathbf{G}_{\mathrm{m}}$ defines a $\mu_{n}$-torsor over $\mathbf{G}_{\mathrm{m}}$. The corresponding element of $H^{1}\left(\mathbf{G}_{\mathrm{m}}, \mu_{n}\right)$ is denoted by $c_{\mathrm{can}, n}$.

Let $D$ be a disk centered at 0 and let $f: D \rightarrow \mathbf{G}_{\mathrm{m}}$ be a nonconstant morphism. Let $c_{n}=f^{*} c_{\text {can }, p^{n}} \in H^{1}\left(D, \mu_{p^{n}}\right)$. Let $Y_{n} \rightarrow D$ be the corresponding $\mu_{p^{n}}$-torsor.

Let $f(X)=\sum_{k \geq 0} a_{k} X^{k}$ be the power series of $f$. Let $e_{0}(f)=\inf \{k \geq 1 \mid$ $\left.a_{k} \neq 0\right\}$ be the ramification index of $f$ at 0 . 
Let $r_{0}\left(c_{n}\right)=\inf \left\{r>0 \mid Y_{n}\right.$ is not split above $\left.b_{0, r}\right\}$ when it exists. Then $Y_{n}$ is trivial above $D\left(0, r_{0}\left(c_{n}\right)^{-}\right)$(otherwise it could be extended to a nontrivial finite cover of $\left.\mathbf{P}^{1}\right)$.

Let $y_{n} \in Y_{n}$ be above $x_{n}=b_{0, r_{0}\left(c_{n}\right)}$. The cover $Y_{n} \rightarrow D$ induces a morphism $\mathcal{H}\left(x_{n}\right) \rightarrow \mathcal{H}\left(y_{n}\right)$ of complete valued fields. Let $k\left(x_{n}\right) \rightarrow k\left(y_{n}\right)$ be the morphism of their residue fields.

We want to study the asymptotic behavior of $r_{0}\left(c_{n}\right)$ as $n \rightarrow \infty$, and the field $k\left(y_{n}\right)$.

Proposition 2.4. Their exists $C$ such that $r_{0}\left(c_{n}\right)=C p^{-n / e_{0}(f)}$ for $n$ large enough. Moreove, for $n$ large enough, $\left[k\left(y_{n}\right): k\left(x_{n}\right)\right]=\left[\mathcal{H}\left(y_{n}\right): \mathcal{H}\left(x_{n}\right)\right]=p$, and $k\left(y_{n}\right)$ is isomorphic to $k(X)[T] /\left(T^{p}-T-X^{e_{0}(f)}\right)$.

Proof. Up to multiplying $f$ by a constant, one can assume $f(0)=1$. Let $N=e_{0}(f)$. Up to replacing $D$ by a smaller disk, one can assume that $\sum_{k \geq N} a_{k} X^{k}$ has an $N$ th root $t$, so that $f=1+t^{N}$. Moreover up to replacing $D$ by a smaller disk, one can assume that $t$ induces an isomorphism $t: D \rightarrow D_{0}$ where $D_{0}$ is also a disk centered at 0 . Since $t$ maps $b_{0, r}$ to $b_{0, \lambda r}$ where $\lambda$ is a constant, it is enough to prove the result for $f t^{-1}$. One can thus assume that $f=1+X^{N}$.

Then $f_{n-1}:=f^{1 / p^{n-1}}=\sum_{k} b_{k} X^{N k}$ where $b_{k}=\left(\begin{array}{c}1 / p^{n-1} \\ k\end{array}\right)$ and

$$
v_{p}\left(b_{k}\right)=-k(n-1)-v_{p}(k !) \text {. }
$$

The series $f^{1 / p^{n-1}}$ is convergent on the disk of radius $p^{-\left(n-1+\frac{1}{p-1}\right) / N}$. By replacing $n-1$ by $n$, one finds that $r_{0}\left(c_{n}\right) \geq \lambda_{n}:=p^{-\left(n+\frac{1}{p-1}\right) / N}$. Let $1+y \in O\left(Y_{n}\right)$ be the $p^{n}$ th root of $f$ such that $y(0)=0$. Then $y$ satisfies the equation

$$
\sum_{k=1}^{p}\left(\begin{array}{l}
p \\
k
\end{array}\right) y^{k}=\sum_{k \geq 1} b_{k} X^{N k} .
$$

Let $b=b_{0, \lambda_{n}} \in D$. Let $b^{\prime}$ be above $b$ in $Y_{n}$ and let $b^{\prime \prime}$ be the image of $b^{\prime}$ in $Y_{n-1}$. Since $\lambda_{n-1}>\lambda_{n}$, the torsor $c_{n-1}$ is split at $b^{\prime \prime}$, and moreover $\left[\mathcal{H}\left(b^{\prime}\right): \mathcal{H}(b)\right]=$ $\left[\mathcal{H}\left(b^{\prime}\right): \mathcal{H}\left(b^{\prime \prime}\right)\right] \mid p$ and $\mathcal{H}(b)=\mathcal{H}\left(b^{\prime}\right) /\left((1+y)^{p}-f_{n-1}\right)$.

At $b$, one has $\left|b_{1} X^{N}\right|_{b}=\left|\frac{1}{p^{n-1}} t^{N}\right|_{b}=p^{-\frac{p}{p-1}}$, and all the other terms in the right member of (2) have smaller norms: $\left|b_{k} t^{N k}\right|_{b}=p^{-\frac{k p}{p-1}+v_{p}(k !)}<p^{-k} \leq p^{-\frac{p}{p-1}}$ for every $k \geq 2$. In particular

$$
\left|\sum_{k=1}^{p}\left(\begin{array}{l}
p \\
k
\end{array}\right) y^{k}\right|_{b^{\prime}}=\left|\sum_{k \geq 1} b_{k} X^{N k}\right|_{b}=p^{-\frac{p}{p-1}} .
$$

If $|y|_{b^{\prime}}<p^{-\frac{1}{p-1}}$, then $\left|\left(\begin{array}{l}p \\ k\end{array}\right) y^{k}\right|_{b^{\prime}}=p^{-1}\left|y^{k}\right|<p^{-\frac{p}{p-1}}$ if $1 \leq k<p$ and $\left|\left(\begin{array}{l}p \\ k\end{array}\right) y^{k}\right|_{b^{\prime}}=$ $|y|^{p}<p^{-\frac{1}{p-1}}$ if $k=p$, which is impossible since $\left|\sum_{k=1}^{p}\left(\begin{array}{l}p \\ k\end{array}\right) y^{k}\right|_{b^{\prime}}=p^{-\frac{p}{p-1}}$. If $|y|_{b^{\prime}}>$ 
$p^{-\frac{1}{p-1}}$, then $\left|\left(\begin{array}{l}p \\ k\end{array}\right) y^{k}\right| b^{\prime}<\left|y^{p}\right|$ for every $1 \leq k<p$ and therefore $\left|\sum_{k=1}^{p}\left(\begin{array}{c}p \\ k\end{array}\right) y^{k}\right|_{b^{\prime}}=$ $\left|y^{p}\right|>p^{-\frac{p}{p-1}}$, which is impossible. Therefore, $|y|_{b^{\prime}}=p^{-\frac{1}{p-1}}$. Hence $|p y|=\left|y^{p}\right|=$ $p^{-\frac{p}{p-1}}$ and $\left|\left(\begin{array}{l}p \\ k\end{array}\right) y^{k}\right|_{b^{\prime}}<p^{-\frac{p}{p-1}}$ for every $2 \leq k \leq p-1$.

Therefore, in the ring $\left\{a \in \mathcal{H}\left(b^{\prime}\right)|| a \mid \leq p^{-\frac{p}{p-1}}\right\} /\left\{a \in \mathcal{H}\left(b^{\prime}\right)|| a \mid<p^{-\frac{p}{p-1}}\right\}$, equation (2) becomes $p y+y^{p}=X^{N} / p^{n-1}$.

Let $z=y / a_{1} \in \mathcal{H}\left(b^{\prime}\right)$ and $u=X / a_{2} \in \mathcal{H}(b) \subset \mathcal{H}\left(b^{\prime}\right)$ where $a_{1}^{p-1}=-p$ and $a_{2}^{N}=p^{n-1} a_{1}^{p}$. One has $|z|_{b^{\prime}}=|u|_{b^{\prime}}=1$ and $k(b)=k(\bar{u})$. Let $\bar{z}$ and $\bar{u}$ be the classes of $z$ and $u$ in $k\left(b^{\prime}\right)$. Equation (2) induces in $k\left(b^{\prime}\right)$ the equality

$$
\bar{z}^{p}-\bar{z}=\bar{u}^{N} .
$$

Therefore $k(b)[\bar{z}] \subset k\left(b^{\prime}\right)$ is a nontrivial extension of $k(b)$. Since $\left[\mathcal{H}\left(b^{\prime}\right): \mathcal{H}(b)\right] \mid p$, one finds that $\left[\mathcal{H}\left(b^{\prime}\right): \mathcal{H}(b)\right]=p, b=x_{n}$, and $k\left(b^{\prime}\right)=k(b)[\bar{z}]$ is the desired Artin-Schreier extension of $k(b)$ (because $k(b)=k(\bar{u})$ ).

If one writes $e_{0}(f)=p^{m} d$ where $d$ is prime to $p$, the genus of the ArtinSchreier curve $T^{p}-T=X^{e_{0}(f)}$ is $g=(d-1)(p-1) / 2$ (cf. [6, §2.2, eq. (8)]). In particular, if $e_{0}(f)$ is not a power of $p$, then, for $n$ large enough, $k\left(y_{n}\right)$ is not isomorphic to $k(X)$, so that if $Y_{n}$ is an analytic domain of a curve $\widetilde{Y}_{n}$, one must have $y_{n} \in V\left(\widetilde{Y}_{n}\right)$. It should be noticed that $e_{0}(f)=\operatorname{ord}_{0}\left(\frac{d f}{f}\right)+1$, where $\operatorname{ord}_{0} \omega$ denotes the $x$-adic valuation of $\frac{d f}{f d X} \in \mathbf{C}_{p}[[X]]$.

\section{§2.3. Resolution of nonsingularities for Mumford curves}

In this subsection we show that Mumford curves over $\overline{\mathbf{Q}}_{p}$ satisfy resolution of nonsingularities.

A proper curve $X$ over $\overline{\mathbf{Q}}_{p}$ is a Mumford curve if the following equivalent properties are satisfied:

- all normalized irreducible components of its stable reduction are isomorphic to $\mathbf{P}^{1}$;

- $X^{\text {an }}$ is locally isomorphic to $\mathbf{P}^{1, \text { an }}$.

The universal topological covering $\Omega$ of $X^{\text {an }}$ for a Mumford curve $X$ is an open subset of $\mathbf{P}^{1, \text { an }}$. More precisely there is a Schottky subgroup $\Gamma$ of $\operatorname{PGL}_{2}\left(\mathbf{C}_{p}\right)$, i.e. a free finitely generated discrete subgroup of $\mathrm{PGL}_{2}\left(\mathbf{C}_{p}\right)$, such that $\Omega=\mathbf{P}^{1 \text {,an }} \backslash \mathcal{L}$ where $\mathcal{L}$ is the closure of the set of $\mathbf{C}_{p}$-points stabilized by some nontrivial element of $\Gamma$. The points of $\mathcal{L}$ are of type 1, i.e. are $\mathbf{C}_{p}$-points. Then $X$ is $p$-adic analytically uniformized as

$$
X^{\text {an }}=\Omega / \Gamma,
$$

and $\Gamma=\pi_{1}^{\mathrm{top}}(X)$. 
Lemma 2.5. Let $I$ be an infinite subset of $K_{0}$, where $K_{0} \subset K$ is a finite extension of $\mathbf{Q}_{p}$. Let $x \in \overline{\mathbf{Q}}_{p}$ be a point not belonging to $I$. Let $E=\left\{k \in \mathbf{N} \mid \exists a \in \mathbf{Q}_{p}^{(I)}, k=\right.$ $\left.\operatorname{ord}_{x}\left(\sum_{i \in I} \frac{a_{i}}{X-i}\right)\right\}$ and $u_{n}=\#(E \cap[0, n])$. Then the sequence $\left(u_{n} / n\right)_{n}$ does not go to 0 as $n \rightarrow \infty$.

Proof. Up to replacing $K_{0}$ by $K_{0}[x]$ and $i$ by $i+x$ for every $i \in I$, one can assume $x=0$. Let $V$ be the $\mathbf{Q}_{p}$-vector subspace of $K_{0}[[X]]$ generated by $\left(\frac{1}{X-i}\right)_{i \in I}$. Let $C=\left[K_{0}: \mathbf{Q}_{p}\right]$.

Let $V_{n}$ be the image of the $\mathbf{Q}_{p}$-linear map $\phi_{n}: V \rightarrow K_{0}[[X]] / X^{n}$. One has $n \in E$ if and only if $\operatorname{Ker}\left(\phi_{n}\right) \subsetneq \operatorname{Ker}\left(\phi_{n-1}\right)$. Therefore

- $\operatorname{dim}_{\mathbf{Q}_{p}} V_{n}=\operatorname{dim}_{\mathbf{Q}_{p}} V_{n-1}$ if $n \notin E$;

- $\operatorname{dim}_{\mathbf{Q}_{p}} V_{n} \leq C+\operatorname{dim}_{\mathbf{Q}_{p}} V_{n-1}$.

Hence $\operatorname{dim}_{\mathbf{Q}_{p}} V_{n} \leq C u_{n}$. However, the morphism $f_{n}: V_{n} \otimes_{\mathbf{Q}_{p}} K \rightarrow K[[X]] / X^{n}$ is surjective. Indeed, let $i_{1}, \ldots, i_{n}$ be $n$ different elements of $I$ and let $\bar{P} \in K[[X]] / X^{n}$. Let $P$ be a representative of $\bar{P}$ in $K[X]$. Let $R$ be the remainder of division of $P \prod_{k=1}^{n}\left(X-i_{k}\right)$ by $X^{n}$. Then, since $\operatorname{deg}(R)<n$,

$$
\frac{R}{\prod_{k}\left(X-i_{k}\right)}=\sum_{k} \frac{a_{k}}{X-i_{k}}
$$

for some $\left(a_{k}\right)_{k}$ in $K^{n}$, and, since $\prod_{k}\left(X-i_{k}\right)$ is invertible in $K[[X]]$,

$$
\bar{P}=f_{n}\left(\sum_{k} \frac{1}{X-i_{k}} \otimes a_{i}\right)
$$

which proves that $f_{n}$ is surjective. Therefore $\operatorname{dim}_{\mathbf{Q}_{p}} V_{n}=\operatorname{dim}_{\mathbf{K}} V_{n} \otimes_{\mathbf{Q}_{p}} K \geq n$ and $u_{n} / n \geq 1 / C$.

Let $I$ be an infinite subset of $K_{0}$, where $K_{0} \subset K$ is a finite extension of $\mathbf{Q}_{p}$. Let $x \in \overline{\mathbf{Q}}_{p}$. Lemma 2.5 shows that there exists $\left(a_{i}\right) \in \mathbf{Q}_{p}^{(I)}$ such that if $g=\sum \frac{a_{i}}{X-i}$ then $\operatorname{ord}_{x}(g)+1$ is not a power of $p$. Up to multiplying all the $a_{i}$ by $p^{\max _{i \in I}\left(-v_{p}\left(a_{i}\right)\right)}$, one can assume $a_{i} \in \mathbf{Z}_{p}$. Let $I_{0} \subset I$ be the support of the family $\left(a_{i}\right)_{i \in I}$.

For $i \in I_{0}$ and $n \geq 0$, let $a_{i, n} \in \mathbf{Z}$ be such that $v_{p}\left(a_{i, n}-a_{i}\right) \geq n$.

Let

$$
f_{n}=\prod_{i \in I_{0}}\left(\frac{X-i}{x-i}\right)^{a_{i, n}}: \mathbf{P}^{1} \backslash I_{0} \rightarrow \mathbf{G}_{\mathrm{m}} .
$$

Let $D=\left\{z \in \mathbf{G}_{\mathrm{m}}|| X-\left.x\right|_{z}<\min _{i \in I_{0}}|x-i|\right\}$. The sequence $\left(f_{n}\right)$ is uniformly convergent on every affinoid subset of $D$ and defines over $D$ a morphism $f$ : $D \rightarrow \mathbf{G}_{\mathrm{m}}$ and $f^{\prime} / f=g$ over $D$. 
Let $c_{n}: Y_{n} \rightarrow \mathbf{P}^{1} \backslash I_{0}$ be the $\mu_{p^{n}}$-torsor over $\mathbf{P}^{1} \backslash I_{0}$ obtained by pulling back along $f_{n}$ the canonical torsor (note that $c_{n}$ only depends on $a_{i}$ and not on $a_{i, n}$ ). The restriction of $c_{n}$ to $D$ is also the pullback of the canonical torsor along $f$.

According to Proposition 2.4, there is a point $y_{n} \in Y_{n}$ such that $g_{k\left(y_{n}\right)} \geq 1$ and $c_{n}\left(y_{n}\right) \rightarrow x$.

One gets the following result:

Proposition 2.6. Let $I$ be an infinite subset of a finite extension of $\mathbf{Q}_{p}$. For every $x \in \overline{\mathbf{Q}}_{p}$, there is a finite subset $I_{0} \subset I \backslash\{x\}$ and a $\mathbf{Z}_{p}(1)$-torsor $c=\left(c_{n}\right.$ : $\left.Y_{n} \rightarrow \mathbf{P}^{1} \backslash I_{0}\right)$ of $\mathbf{P}^{1} \backslash I_{0}$ and for every $n \geq 1$ a point $y_{n} \in Y_{n}$ such that $g_{k\left(y_{n}\right)} \geq 1$ and $c_{n}\left(y_{n}\right) \rightarrow x$.

Theorem 2.7. Let $X$ be a Mumford curve over $\overline{\mathbf{Q}}_{p}$. Then $X$ satisfies resolution of nonsingularities.

Proof. Let $x \in X\left(\overline{\mathbf{Q}}_{p}\right)$. According to Proposition 2.2, it is enough to show that $x \in \bar{V}(X)$.

Let $\Omega=\mathbf{P}^{1 \text {,an }} \backslash \mathcal{L}$ be the topological universal cover of $X$, and let $\Gamma=$ $\operatorname{Gal}(\Omega / X) \subset \mathrm{PGL}_{2}\left(\mathbf{C}_{p}\right)$. Let $z$ be a point of $\Omega$ above $x$. One can assume that $\Gamma \subset \mathrm{PGL}_{2}\left(K_{0}\right)$, where $K_{0}$ is a finite extension of $\mathbf{Q}_{p}$. Let $g \in \Gamma$ and $t \in \mathcal{L} \cap \mathbf{P}^{1}\left(\overline{\mathbf{Q}}_{p}\right)$ a point that is not fixed by $g$. Up to replacing $K_{0}$ by a finite extension, one can assume $t \in K_{0}$. Let $I=\left\{g^{n}(t)\right\}_{n \in \mathbf{Z}} \subset K_{0}$. According to Proposition 2.6, there exists a finite subset $I_{0}$ of $I$ and a $\mathbf{Z}_{p}(1)$-torsor $\left(c_{n}: Y_{n} \rightarrow \mathbf{P}^{1} \backslash I_{0}\right)$ of $\mathbf{P}^{1} \backslash I_{0}$ and a point $y_{n}$ of $Y_{n}$ such that $g_{k_{y_{n}}} \geq 1$ and $z_{n}:=c_{n}\left(y_{n}\right) \rightarrow z$. Let $x_{n}$ be the image of $z_{n}$ in $X$. Fix $n$. We will show that $x_{n} \in \widetilde{V}(X)$. Let $\epsilon$ be small enough so that

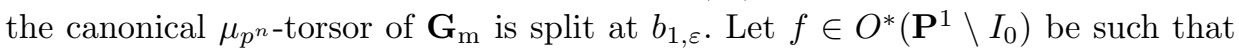
$c_{n}=f^{*} \mu_{p^{n}}$.

Lemma 2.8. Let $f \in O^{*}(\Omega)$. There exists a family $\left(f_{\Gamma^{\prime}}\right)_{\Gamma^{\prime}}$ of elements of $O^{*}(\Omega)$, where $\Gamma^{\prime}$ runs through the filtered set of subgroups of $\Gamma$ of finite index, such that:

- for every $g \in \Gamma^{\prime}, \frac{f_{\Gamma^{\prime}} \circ g}{f_{\Gamma^{\prime}}}$ is a constant function, i.e. $f_{\Gamma^{\prime}}$ is a theta function of $X / \Gamma^{\prime}$;

- $\left(f_{\Gamma^{\prime}}\right)_{\Gamma^{\prime}}$ converges uniformly to $f$ on every affinoid domain of $\Omega$.

Proof. The subgroup of $O^{*}(\Omega)$ generated by the constants and functions of the form $\frac{z-a}{z-b}$, where $a$ and $b$ are the zeros of a nontrivial element of $\Gamma$, is dense in $O^{*}(\Omega)$. Therefore one can assume that $f=\frac{z-a}{z-b}$ and that the subgroup $H$ of elements of $\Gamma$ fixing $a$ and $b$ is nontrivial. Let $z_{0} \in \Omega\left(\mathbf{C}_{p}\right)$. Then

$$
f_{\Gamma^{\prime}}=f\left(z_{0}\right) \prod_{g \in\left(H \cap \Gamma^{\prime}\right) \backslash \Gamma^{\prime}} \frac{f(g(z))}{f\left(g\left(z_{0}\right)\right)}
$$

has the desired properties. 
Apply Lemma 2.8 to $f$; let $\left(f_{\Gamma^{\prime}}\right)$ be as in the lemma. In particular, there exists a subgroup $\Gamma^{\prime}$ of finite index of $\Gamma$ such that $\left|f_{\Gamma^{\prime}} / f-1\right|_{z_{n}}<\varepsilon$. Let $c^{\prime}=f_{\Gamma^{\prime}}^{*} \mu_{p^{n}}$ : $Y^{\prime} \rightarrow \Omega$. Then $c_{n}-c^{\prime}$ is split at $z_{n}$. Therefore $c_{n}$ and $c^{\prime}$ are isomorphic above $z_{n}$ and there is $y^{\prime} \in Y^{\prime}$ above $z_{n}$ such that $g_{k_{y_{n}^{\prime}}} \geq 1$. Since $f_{\Gamma^{\prime}}$ is a theta function of

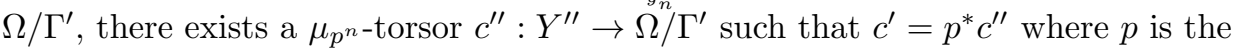
topological cover $\Omega \rightarrow \Omega / \Gamma^{\prime}$. Since $Y^{\prime} \rightarrow Y^{\prime \prime}$ is a topological cover, the image $y^{\prime \prime}$ of $y^{\prime}$ in $Y^{\prime \prime}$ is in $V\left(Y^{\prime \prime}\right)$. Since $Y^{\prime \prime} \rightarrow X$ is a finite cover and maps $y^{\prime \prime}$ to $x_{n}$, one has $x_{n} \in \tilde{V}(X)$. Therefore $x \in \bar{V}(X)$.

Since $\mathbf{P}^{1} \backslash\{0,1, \infty\}$ and punctured Tate curves have finite étale covers that are nonempty Zariski open subsets of Mumford curves, they also satisfy resolution of nonsingularities. For every smooth connected curve $X$ over $\overline{\mathbf{Q}}_{p}$, there exists a nonempty Zariski open subset $U \subset X$ and a finite étale cover $U \rightarrow V=$ $\mathbf{P}^{1} \backslash\left\{x_{1}, \ldots, x_{n}\right\}$ with $n \geq 3$. Since $\mathbf{P}^{1} \backslash\left\{x_{1}, \ldots, x_{n}\right\}$ satisfies resolution of nonsingularities, so does $U$. Indeed, if $u \in U^{\text {an }}$ is a point of type 2 and $v$ is its image in $V$, let $Y \rightarrow V$ be a finite étale cover such that $v$ is in the image of $V(Y)$. Up to replacing $Y$ by a bigger cover, one can assume that $Y$ is Galois over $V$, so that every preimage of $v$ is in $V(Y)$, and there is a morphism $Y \rightarrow U$ of $V$-covers; $u$ is then in the image of $V(Y)$. The same argument also works for $\mathbf{C}_{p}$-curves.

Corollary 2.9. Every $\overline{\mathbf{Q}}_{p}$-curve (resp. $\mathbf{C}_{p}$-curve) has a Zariski dense open subset which satisfies resolution of nonsingularities.

\section{$\S 3$. Resolution of nonsingularities and anabelian tempered geometry}

\section{§3.1. Tempered fundamental group}

Let $K$ be a complete nonarchimedean field. A morphism $f: S^{\prime} \rightarrow S$ of $K$-analytic spaces is said to be an étale cover if $S$ is covered by open subsets $U$ such that $f^{-1}(U)=\coprod V_{j}$ and $V_{j} \rightarrow U$ is étale finite $([5])$.

For example, finite étale covers, also called algebraic covers, and covers in the usual topological sense for the Berkovich topology, also called topological covers, are étale covers.

Then, André defines tempered covers as follows:

Definition 3.1 ([1, Def. 2.1.1]). An étale cover $S^{\prime} \rightarrow S$ is tempered if it is a quotient of the composition of a topological cover $T^{\prime} \rightarrow T$ and of a finite étale cover $T \rightarrow S$.

This is equivalent to saying that the cover becomes a topological cover after pullback along some finite étale cover. 
We denote by $\operatorname{Cov}^{\text {temp }}(X)$ (resp. $\left.\operatorname{Cov}^{\text {alg }}(X), \operatorname{Cov}^{\text {top }}(X)\right)$ the category of tempered covers (resp. algebraic covers, topological covers) of $X$ (with the obvious morphisms).

A geometric point of a $K$-manifold $X$ is a morphism of Berkovich spaces $\mathcal{M}(\Omega) \rightarrow X$ where $\Omega$ is an algebraically closed complete isometric extension of $K$.

Let $\bar{x}$ be a geometric point of $X$. Then one has a functor

$$
F_{\bar{x}}: \operatorname{Cov}^{\text {temp }}(X) \rightarrow \text { Set }
$$

which maps a cover $S \rightarrow X$ to the set $S_{\bar{x}}$. If $\bar{x}$ and $\bar{x}^{\prime}$ are two geometric points, then $F_{\bar{x}}$ and $F_{\bar{x}^{\prime}}$ are (noncanonically) isomorphic ([5, Prop. 2.9]).

A functor $F: \operatorname{Cov}^{\text {temp }}(X) \rightarrow$ Set is said to be a fiber functor if it is isomorphic to $F_{\bar{x}}$ for some (and therefore every) geometric point $\bar{x}$ of $X$.

Proposition 3.2. A fiber functor of $\operatorname{Cov}^{\text {temp }}(X)$ is pro-representable.

If $F$ is a fiber functor of $\mathrm{Cov}^{\text {temp }}(X)$, a pointed tempered cover of $X$ is a couple $(S, s)$ where $S$ is a tempered cover of $X$ and $s \in F(S)$. A pro-tempered cover $\tilde{X}$ of $X$ is called universal if $F_{\widetilde{X}}:=\operatorname{Hom}(\widetilde{X}$,$) is a fiber functor of \operatorname{Cov}^{\text {temp }}(X)$.

The tempered fundamental group of $X$ pointed at a fiber functor $F$ is

$$
\pi_{1}^{\text {temp }}(X, F)=\text { Aut } F
$$

The tempered fundamental group of $X$ pointed at a universal pro-tempered cover $\widetilde{X}$ is

$$
\pi_{1}^{\text {temp }}(X, \tilde{X})=\text { Aut } \tilde{X}=\text { Aut } F_{\tilde{X}} .
$$

The tempered fundamental group of $X$ pointed at a geometric point $\bar{x}$ is

$$
\pi_{1}^{\mathrm{temp}}(X, \bar{x})=\pi_{1}^{\mathrm{temp}}\left(X, F_{\bar{x}}\right) .
$$

When $X$ is a smooth algebraic $K$-variety, $\operatorname{Cov}^{\text {temp }}\left(X^{\text {an }}\right)$ and $\pi_{1}^{\text {temp }}\left(X^{\text {an }}, F\right)$ will also be denoted simply by $\operatorname{Cov}^{\text {temp }}(X)$ and $\pi_{1}^{\text {temp }}(X, F)$.

By considering the stabilizers $\left(\operatorname{Stab}_{F(S)}(s)\right)_{(S, s)}$ as a basis of open subgroups of $\pi_{1}^{\text {temp }}(X, F)$, the latter becomes a topological group. It is a pro-discrete topological group.

If $X$ is algebraic, and if $K$ is of characteristic zero and has only countably many finite extensions in a fixed algebraic closure $\bar{K}$, then $\pi_{1}^{\text {temp }}(X, F)$ has a countable fundamental system of neighborhoods of 1 and all its discrete quotient groups are finitely generated ([1, Prop. 2.1.7]).

Thus, as usual, the tempered fundamental group depends on the basepoint only up to inner automorphism (this topological group, considered up to conjugation, will sometimes be denoted simply by $\left.\pi_{1}^{\text {temp }}(X)\right)$. 
The full subcategory of tempered covers $S$ for which $F_{\bar{x}}(S)$ is finite is equivalent to $\operatorname{Cov}^{\text {alg }}(S)$, hence

$$
\left.\pi_{1}^{\mathrm{temp}(X}, \bar{x}\right)=\pi_{1}^{\operatorname{alg}}(X, \bar{x})
$$

(here and in what follows, ${ }^{\wedge}$ denotes profinite completion).

For any morphism $X \rightarrow Y$, the pullback defines a functor $\operatorname{Cov}^{\text {temp }}(Y) \rightarrow$ $\operatorname{Cov}^{\text {temp }}(X)$. If $\bar{x}$ is a geometric point of $X$ with image $\bar{y}$ in $Y$, this gives rise to a continuous homomorphism

$$
\pi_{1}^{\mathrm{temp}}(X, \bar{x}) \rightarrow \pi_{1}^{\mathrm{temp}}(Y, \bar{y})
$$

(hence an outer morphism $\pi_{1}^{\text {temp }}(X) \rightarrow \pi_{1}^{\text {temp }}(Y)$ ).

One has the analog of the usual Galois correspondence:

Theorem 3.3 ([1, Th. 1.4.5]). A fiber functor $F$ induces an equivalence of categories between the category of tempered covers of $X$ and the category $\pi_{1}^{\mathrm{temp}}(X, F)$-Set of discrete sets endowed with an action of $\pi_{1}^{\mathrm{temp}}(X, F)$ that factorizes through a finite quotient.

If $S$ is a finite Galois cover of $X$, its universal topological cover $S^{\infty}$ is still Galois and every connected tempered cover is dominated by such a Galois tempered cover.

Let $\bar{x}$ be a geometric point of $X$. Let "lim ${ }_{I} "\left(S_{i}, s_{i}\right)$ be a pointed universal pro-finite cover of $X$. Let $\left(S_{i}^{\infty}, s_{i}^{\infty}\right)$ be the pointed universal topological cover of $\left(S_{i}, s_{i}\right)$. Then "lim ${ }_{I} S_{i}^{\infty}$ is a universal pro-tempered cover of $X$.

If $S=$ "lim" $S_{i}$ is a pro-tempered cover of $X$, we denote by $|S|$ the topological space $\lim _{\leftarrow} S_{i}$.

Let $(\bar{X}, D)$ be a marked curve. Let $X=\bar{X} \backslash D$. If $S$ is a tempered cover of $X$, it extends uniquely, and functorially in $Y$, to a ramified cover $\bar{S} \rightarrow \bar{X}$ ([1, Th. III.2.1.11]). If $S=" \lim S_{i}$ " is a pro-tempered cover of $X$, we denote by $|S|_{c}$ the topological space $\lim \overleftarrow{S}_{i}$. Any morphism $S \rightarrow S^{\prime}$ of pro-tempered covers induces a continuous map $|S|_{c} \rightarrow\left|S^{\prime}\right|_{c}$. In particular, if $\widetilde{X}$ is a universal pro-tempered cover of $X$, then $\pi_{1}^{\text {temp }}(X, \tilde{X})=$ Aut $\tilde{X}$ acts on $|\widetilde{X}|_{c}$.

Let $\mathcal{P}$ be the set of prime numbers and let $\mathbb{L}$ be a subset of $\mathcal{P}$. We define an $\mathbb{L}$-integer to be a integer which is a product of elements of $\mathbb{L}$. We write $\left(p^{\prime}\right)$ for $\mathcal{P} \backslash\{p\}$.

An $\mathbb{L}$-tempered cover $S$ of $X$ is a tempered cover such that there exists a finite étale Galois cover $Y \rightarrow X$ of index an $\mathbb{L}$-integer such that $S \times_{X} Y \rightarrow Y$ is a topological cover. We denote by $\operatorname{Cov}^{\text {temp }}(X)^{\mathbb{L}}$ the category of $\mathbb{L}$-tempered covers. If $F$ is a fiber functor of $\operatorname{Cov}^{\text {temp }}(X)$, we denote by $\pi_{1}^{\text {temp }}(X, F)^{\mathbb{L}}$ the topological 
group of automorphisms of $F_{\mid} \operatorname{Cov} \operatorname{temp}(X)^{\mathrm{L}}$. If $\tilde{X}$ is a universal pro-tempered cover of $X$, we define $\widetilde{X}^{\mathbb{L}}=" \lim _{\tilde{X} \rightarrow Y} " Y$ where $Y$ runs over $\widetilde{X}$-pointed $\mathbb{L}$-tempered covers.

If $\mathbb{L} \subset \mathbb{L}^{\prime}$, the fully faithful functor $\operatorname{Cov}^{\text {temp }}(X)^{\mathbb{L}} \rightarrow \operatorname{Cov}^{\text {temp }}(X)^{\mathbb{L}^{\prime}}$ induces a morphism $\pi_{1}^{\text {temp }}(X, F)^{\mathbb{L}^{\prime}} \rightarrow \pi_{1}^{\text {temp }}(X, F)^{\mathbb{L}}$, and in particular when $\mathbb{L}^{\prime}=\mathcal{P}$, one gets a morphism $\pi_{1}^{\text {temp }}(X, F) \rightarrow \pi_{1}^{\text {temp }}(X, F)^{\mathbb{L}}$.

\section{§3.2. Decomposition group of a point}

Recall the following notation: if $X=\bar{X} \backslash D$ is a hyperbolic curve, then $\mathbb{G}_{X}$ is the dual graph of the stable reduction of $(\bar{X}, D)$, and $\mathbb{T}_{X}$ is the universal cover of $\mathbb{G}_{X}$. Moreover, if $\mathbb{G}$ is a graph, then $V(\mathbb{G})$ denotes the set of vertices of $\mathbb{G}$.

Let $(\bar{X}, D)$ be a hyperbolic marked $K$-curve, and let $X=\bar{X} \backslash D$. Let $\tilde{X}$ be a universal pro-tempered cover. We define $\widetilde{X}^{\mathbb{L}}=\varliminf_{\varliminf_{Y}} Y^{\infty}$ where $Y$ runs over the $\widetilde{X}$-pointed finite étale covers of $X$ of index an $\mathbb{L}$-integer, and $Y^{\infty}$ is the $\widetilde{X}$-pointed universal topological cover of $Y$. If $p \notin \mathbb{L}$, every morphism $Z \rightarrow Y$ of $\widetilde{X}$-pointed finite étale covers of $X$ of index an $\mathbb{L}$-integer induces a morphism of trees $\mathbb{T}_{Z} \rightarrow \mathbb{T}_{Y}$; we define $\mathbb{T}_{X}^{\mathbb{L}}$ to be $\lim _{Y} \mathbb{T}_{Y}$. The family of embeddings $V\left(\mathbb{T}_{Y}\right) \rightarrow Y^{\infty}$ induces an embedding $V\left(\mathbb{T}_{X}^{\mathbb{L}}\right) \rightarrow\left|\widetilde{X}^{\mathbb{L}}\right|$.

Let $z=\left(z_{Y}\right)_{Y}$ be an edge of $\mathbb{T}_{X}^{\mathbb{L}}$. Let $\left(\overline{\mathcal{Y}}, \mathcal{D}_{Y}\right)$ be the stable model of $Y$ and let $\tilde{z}_{Y}$ be the node of $\overline{\mathcal{Y}}_{k}$ corresponding to the image of $z_{Y}$ in $\mathbb{G}_{Y}$. Let $S_{z_{Y}}=S(\overline{\mathcal{Y}}) \cap$ $\pi_{\bar{Y}}^{-1}\left(\tilde{z}_{Y}\right)$, where $\pi_{\overline{\mathcal{Y}}}$ is the specialization map $\bar{Y}^{\text {an }} \rightarrow \overline{\mathcal{Y}}_{k}$. Then every morphism $Y \rightarrow Y^{\prime}$ induces a homeomorphism $S_{z_{Y}} \rightarrow S_{z_{Y^{\prime}}}$ and we define $S_{z}=\lim _{Y_{Y}} S_{z_{Y}}$.

Then $\pi_{1}^{\text {temp }}(X, \widetilde{X})$ acts on $|\widetilde{X}|_{c}$. Similarly $\pi_{1}^{\text {temp }}(X, \widetilde{X})^{\mathbb{L}}$ acts on $|\widetilde{X}|_{c}^{\mathbb{L}}$. Let $x \in|\widetilde{X}|_{c}$. We denote by $D_{x}$ the stabilizer of $x$ in $\pi_{1}^{\text {temp }}(X, \widetilde{X})$, and by $D_{x, \mathbb{L}}$ its image in $\pi_{1}^{\text {temp }}(X, \widetilde{X})^{\mathbb{L}}$. The group $D_{x, \mathbb{L}}$ is the stabilizer of the image of $x$ in $\left|\widetilde{X}^{\mathbb{L}}\right|_{c}$. The decomposition group depends only on the image of $x$ in $\bar{X}$ up to conjugacy.

Theorem 3.4 ([11, Cor. 3.11]). If $X_{\alpha}$ and $X_{\beta}$ are two hyperbolic $\overline{\mathbf{Q}}_{p}$-curves, then every (outer) isomorphism $\gamma: \pi_{1}^{\text {temp }}\left(X_{\alpha, \mathbb{C}_{p}}\right)^{\left(p^{\prime}\right)} \simeq \pi_{1}^{\text {temp }}\left(X_{\beta, \mathbb{C}_{p}}\right)^{\left(p^{\prime}\right)}$ determines, functorially in $\gamma$, an isomorphism of graphs $\bar{\gamma}: \mathbb{G}_{X_{\alpha}} \simeq \mathbb{G}_{X_{\beta}}$.

More precisely, the map $x \mapsto D_{x,\left(p^{\prime}\right)}$ identifies the vertices of $\mathbb{T}_{X}^{\left(p^{\prime}\right)}$ with the maximal compact subgroups of $\pi_{1}^{\text {temp }}(X)^{\left(p^{\prime}\right)}$, and two vertices $x, x^{\prime}$ of $\mathbb{T}_{X}^{\left(p^{\prime}\right)}$ are linked by an edge if and only if $D_{x,\left(p^{\prime}\right)} \cap D_{x^{\prime},\left(p^{\prime}\right)} \neq\{1\}$. Therefore the isomorphism $\gamma: \pi_{1}^{\text {temp }}\left(X_{\alpha, \mathbb{C}_{p}}\right)^{\left(p^{\prime}\right)} \simeq \pi_{1}^{\text {temp }}\left(X_{\beta, \mathbb{C}_{p}}\right)^{\left(p^{\prime}\right)}$ induces an equivariant isomorphism of graphs $\mathbb{T}_{X_{\alpha}}^{\left(p^{\prime}\right)} \rightarrow \mathbb{T}_{X_{\beta}}^{\left(p^{\prime}\right)}$, which gives $\bar{\gamma}$ by quotienting by the action of the tempered fundamental group. 
Proposition 3.5. Let $x_{1} \neq x_{2} \in \tilde{V}(\widetilde{X})$. Then $D_{x_{1}}$ and $D_{x_{2}}$ are not commensurable.

Proof. Let $(Y, f: \widetilde{X} \rightarrow Y)$ be a pointed finite étale cover of $X$ such that $f\left(x_{1}\right) \neq$ $f\left(x_{2}\right) \in V(Y)$. Then $D_{x_{i}} \cap \pi_{1}^{\text {temp }}(Y, \widetilde{X})=D_{f\left(x_{1}\right)}$. But the images of $D_{f\left(x_{1}\right)}$ and $D_{f\left(x_{2}\right)}$ in $\pi_{1}^{\text {temp }}(Y, \widetilde{X})^{\left(p^{\prime}\right)}$ are already not commensurable.

Corollary 3.6. Let $x \in \widetilde{V}(\widetilde{X})$. Then $D_{x}$ is its own normalizer.

Proof. Let $g$ be in the normalizer of $D_{x}$. Then $D_{g(x)}=g D_{x} g^{-1}=D_{x}$. Since $x, g(x) \in \widetilde{V}(\widetilde{X})$, Proposition 3.5 tells us that $x=g(x)$, i.e. $g \in D_{x}$.

According to [7, Prop. 10], if $D$ is a compact subgroup of $\pi_{1}^{\text {temp }}(X, \bar{X})$ which is not a pro- $p$ group, there exists $x \in|\widetilde{X}|_{c}$ such that $D \subset D_{x}$. Moreover, a point $x \in|\widetilde{X}|_{c}$ is in $\widetilde{V}(\widetilde{X})$ if and only if there exists an open finite index subgroup $H \subset$ $\pi_{1}^{\text {temp }}(X)$ such that the image of $D_{x} \cap H$ in $H^{\left(p^{\prime}\right)}$ is noncommutative. Therefore, the set $\widetilde{V}(\widetilde{X})$ can be identified with the set of conjugacy classes of maximal compact subgroups $D$ of $\pi_{1}^{\text {temp }}(X)$ such that the image of $D \cap H$ in $H^{\left(p^{\prime}\right)}$ is noncommutative for some open finite index subgroup $H$ of $\pi_{1}^{\text {temp }}(X)$.

\section{§3.3. Tempered theoreticness of Berkovich topology}

Let $(\bar{X}, D)$ be a $\overline{\mathbf{Q}}_{p}$-marked curve and let $X=\bar{X} \backslash D$. If $Y \rightarrow X$ is a Galois finite étale cover and $\left(\overline{\mathcal{Y}}, \mathcal{D}_{Y}\right)$ is the stable model of $Y$, then $\mathcal{X}_{Y}:=\overline{\mathcal{Y}} / \operatorname{Gal}(Y / X)$ is a semistable model of $\bar{X}$.

Let us say that a topological group is temp-like if it is isomorphic to the tempered fundamental group of a hyperbolic curve over $\overline{\mathbf{Q}}_{p}$ that satisfies resolution of nonsingularities. We will construct, for any temp-like topological group $\Pi$, a topological space $\widetilde{S}(\Pi)$ endowed with a continuous action of $\Pi$. The construction will be purely group-theoretic, so that it will be functorial with respect to isomorphism of topological groups. Moreover when $\Pi=\pi_{1}^{\text {temp }}(X, \widetilde{X})$, we will get a $\pi_{1}^{\text {temp }}(X, \widetilde{X})$-equivariant homeomorphism $|\widetilde{X}| \rightarrow \widetilde{S}\left(\pi_{1}^{\text {temp }}(X, \widetilde{X})\right)$.

Let $\Pi$ be a temp-like topological group. We fix an isomorphism $\Pi \simeq$ $\pi_{1}^{\text {temp }}(X, \widetilde{X})$ (but we will take care that the construction of $\widetilde{S}(\Pi)$ does not depend on this isomorphism). Then there is a smallest normal open subgroup $\Pi^{\infty}$ such that $\Pi / \Pi^{\infty}$ is torsionfree. One can also see $\Pi^{\infty}$ as the closed subgroup generated by the compact subgroups of $\Pi$. One defines the topological group $\Pi^{\left(p^{\prime}\right)}:=\varliminf_{N} \Pi / N^{\infty}$ where $N$ runs through open normal subgroups of $\Pi$ of finite index prime to $p$ (such an $N$ is also temp-like, so that $N^{\infty}$ is well defined). The morphism $\Pi \rightarrow \Pi^{\left(p^{\prime}\right)}$ has dense image. We denote by $\Pi^{\left(p^{\prime}\right), \infty}$ the kernel of $\Pi^{\left(p^{\prime}\right)} \rightarrow \Pi / \Pi^{\infty}$.

If $H$ is a normal open subgroup of $\Pi$ of finite index, let $\widetilde{V}(\Pi)_{H}$ be the set of maximal compact subgroups $D$ of $\Pi$ such that $D \cap H$ is not commutative. Let 
$\widetilde{V}(\Pi)=\bigcup_{H} \tilde{V}(\Pi)_{H}$. The group $\Pi$ acts by conjugacy on $\widetilde{V}(\Pi)$ and on $\widetilde{V}(\Pi)_{H}$ for every $H$.

Recall that there is an equivariant bijection $\widetilde{V}(\widetilde{X}) \rightarrow \widetilde{V}(\Pi)$ that maps $x$ to $D_{x}$. More precisely, if $(Y, f: \widetilde{X} \rightarrow Y)$ is the pointed finite étale cover of $X$ corresponding to $H$, it induces a bijection $\{x \in|\widetilde{X}|: f(x) \in V(Y)\} \rightarrow \widetilde{V}(\Pi)_{H}$. By quotienting by $H^{\infty}$, one gets a bijection $V(Y)^{\infty} \rightarrow \widetilde{V}(\Pi)_{H} / H^{\infty}$, where $V(Y)^{\infty}$ is the preimage of $V(Y)$ under the map $Y^{\infty} \rightarrow Y^{\text {an }}$.

Let $H \subset \Pi$ be a normal open subgroup of finite index. Then $H$ is also templike: it is isomorphic to $\pi_{1}^{\text {temp }}(Y, \widetilde{X})$ for some Galois finite étale cover $Y$ of $X$. Let $V(H)^{\left(p^{\prime}\right)}$ be the set of maximal compact subgroups of $H^{\left(p^{\prime}\right)}$. Let $E(H)^{\left(p^{\prime}\right)}$ be the set of pairs of elements $\left(D, D^{\prime}\right)$ of $V(H)^{\left(p^{\prime}\right)}$ such that $D \cap D^{\prime} \neq\{1\}$. These data define a graph $\mathbb{G}(H)^{\left(p^{\prime}\right)}$. Since $H$ is normal in $\Pi$, the group $\Pi$ acts on $H^{\left(p^{\prime}\right)}$ by conjugacy and therefore on $\mathbb{G}(H)^{\left(p^{\prime}\right)}$. Note that $H^{\left(p^{\prime}\right)}$ also acts by conjugacy on $\mathbb{G}(H)^{\left(p^{\prime}\right)}$ and that the actions of $\Pi$ and of $H^{\left(p^{\prime}\right)}$ coincide on $H$.

According to [7, Th. 6], there is a П-equivariant isomorphism $\mathbb{T}_{Y}^{\left(p^{\prime}\right)} \simeq \mathbb{G}(H)^{\left(p^{\prime}\right)}$ that maps a vertex $x$ to its stabilizer $D_{x}$ under the action of $H^{\left(p^{\prime}\right)}$. If $e=\left(D, D^{\prime}\right) \in$ $E(H)^{\left(p^{\prime}\right)}$, we denote $D_{e}:=D \cap D^{\prime} \subset H^{\left(p^{\prime}\right)}$. The group $D_{e}$ is the stabilizer of the image of $e$ in $\mathbb{T}_{Y}^{\left(p^{\prime}\right)}$ for the action of $H^{\left(p^{\prime}\right)}$ on $\mathbb{G}(H)^{\left(p^{\prime}\right)}$.

Let

$$
\mathbb{G}(H)=\mathbb{G}(H)^{\left(p^{\prime}\right)} / H^{\left(p^{\prime}\right)} \text { and } \quad \mathbb{G}(H)^{\infty}=\mathbb{G}(H)^{\left(p^{\prime}\right)} / H^{\left(p^{\prime}\right), \infty} .
$$

Then $\mathbb{G}(H)$ can be identified with $\mathbb{G}_{Y}$, and $\mathbb{G}(H)^{\infty}$ with $\mathbb{T}_{Y}$.

If $D \in \widetilde{V}(\Pi)_{H}$, the image of $D \cap H$ in $H^{\left(p^{\prime}\right)}$ is a maximal compact subgroup, and therefore defines an element of $V(H)^{\left(p^{\prime}\right)}$, hence a $\Pi$-equivariant map $p_{H}$ : $\widetilde{V}(\Pi)_{H} \rightarrow V(H)^{\left(p^{\prime}\right)}$.

Moreover, the induced map $p_{H}^{\infty}: \widetilde{V}(\Pi)_{H} / H^{\infty} \rightarrow V(H)^{\left(p^{\prime}\right)} / H^{\left(p^{\prime}\right), \infty}$ is bijective: indeed, the diagram

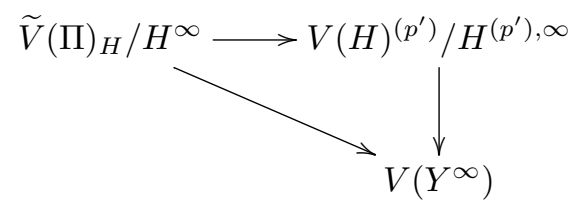

is commutative and the two vertical maps are bijections.

One has $H^{\infty} \subset \Pi^{\infty}$ and one gets a $\Pi$-equivariant map $\iota_{H, \Pi}^{\left(p^{\prime}\right)}: H^{\left(p^{\prime}\right)} \rightarrow \Pi^{\left(p^{\prime}\right)}$.

Lemma 3.7. Let $D \in V(H)^{\left(p^{\prime}\right)}$. The subgroup $\iota_{H, \Pi}^{\left(p^{\prime}\right)}(D) \subset \Pi^{\left(p^{\prime}\right)}$ is either:

- an open subgroup of $D_{1}$ for a unique $D_{1} \in V(\Pi)^{\left(p^{\prime}\right)}$;

- an open subgroup of $D_{e}$ for a unique e $\in \bar{E}(\Pi)^{\left(p^{\prime}\right)}$; or

- $\{1\}$. 
Let $e \in E(H)^{\left(p^{\prime}\right)}$. The subgroup $\iota_{H, \Pi}^{\left(p^{\prime}\right)}\left(D_{e}\right) \subset \Pi^{\left(p^{\prime}\right)}$ is either:

- an open subgroup of $D_{e}$ for a unique $e \in \bar{E}(\Pi)^{\left(p^{\prime}\right)}$; or

- $\{1\}$.

Proof. Uniqueness is clear.

Since $p_{H}^{\infty}$ is surjective, up to conjugating $D$ by an element of $H^{\left(p^{\prime}\right)}$, one can assume that $D$ is the image of $p_{H}$. Let $D_{0} \in \tilde{V}(\Pi)_{H}$ be a preimage of $D$ and let $x$ be the corresponding point of $\widetilde{V}(\widetilde{X})$. Since $H \cap D_{x}$ is open in $D_{x}, \iota_{H, \Pi}^{\left(p^{\prime}\right)}(D)$ is open in the image $D_{x,\left(p^{\prime}\right)}$ of $D_{x}$ under $\Pi \rightarrow \Pi^{\left(p^{\prime}\right)}$. Let $\tilde{x}$ be the image of $x$ in $\widetilde{X}^{\left(p^{\prime}\right)}$. If $\tilde{x} \in V\left(\widetilde{X}^{\left(p^{\prime}\right)}\right)$, then $D_{x,\left(p^{\prime}\right)} \in V(\Pi)^{\left(p^{\prime}\right)}$; if $\tilde{x}$ lies on an edge $e$ of $\mathbb{T}_{X}^{\left(p^{\prime}\right)}$, then $D_{x,\left(p^{\prime}\right)}=D_{e}$; otherwise, the image of $x$ in $X$ lies in a disk and since every prime-to- $p$ cover of a disk is trivial, $D_{x,\left(p^{\prime}\right)}=\{1\}$.

If $e \in E(H)^{\left(p^{\prime}\right)}$, there exists $\bar{y} \in\left|\tilde{Y}^{\left(p^{\prime}\right)}\right|$ such that $D_{e}=D_{x}$. Up to conjugating $D$ by an element of $H^{\left(p^{\prime}\right)}$, one can assume that there is $x \in|\widetilde{X}|$ which maps to $\bar{y}$. Let $\bar{x}$ be the image of $\bar{y}$ in $\left|\widetilde{X}^{\left(p^{\prime}\right)}\right|$. Once again, $\iota_{H, \Pi}^{\left(p^{\prime}\right)}\left(D_{e}\right)$ is open in $D_{\bar{x}}$. Since $\bar{y} \notin V(Y)^{\left(p^{\prime}\right)}$, one has $\bar{x} \notin V(X)^{\left(p^{\prime}\right)}$. Therefore either $\bar{x}$ lies on an edge $e^{\prime}$ of $\mathbb{T}_{X}^{\left(p^{\prime}\right)}$ and $D_{\bar{x}}=D_{e^{\prime}}$, or $x$ lies outside the image of $S(X)^{\left(p^{\prime}\right)}$ and $D_{\bar{x}}=\{1\}$.

We denote $\mathbb{G}_{\Pi^{\infty}}(H)=\mathbb{G}(H)^{\infty} / \Pi^{\infty}$. If one identifies $\mathbb{G}(H)^{\infty}$ with $\mathbb{T}_{Y}$, then $\mathbb{G}_{\Pi \infty}(H)=\mathbb{T}\left(\mathcal{X}_{Y}\right)$. The bijection $p_{H}^{\infty,-1}$ induces a bijection $V_{\Pi \infty}(H) \rightarrow$ $\widetilde{V}(\Pi)_{H} / \Pi^{\infty}$. If $H^{\prime} \subset H$ are two normal subgroups of $\Pi$, the $\Pi$-equivariant injective map $\tilde{V}(\Pi)_{H} \rightarrow \widetilde{V}(\Pi)_{H}^{\prime}$ induces an injective map $V_{\Pi \infty}(H) \rightarrow V_{\Pi \infty}\left(H^{\prime}\right)$.

Let $e=\left(D_{1}, D_{2}\right) \in E(\Pi)^{\infty}$. We denote

$$
\widetilde{A}_{e, H}^{\left(p^{\prime}\right)}:=\left\{D \in V(H)^{\left(p^{\prime}\right)} \mid \exists \tilde{e} \in E(\Pi)^{\left(p^{\prime}\right)},[\tilde{e}]=e \text { and }\{1\} \neq \iota_{H, \Pi}^{\left(p^{\prime}\right)}(D) \subset D_{\tilde{e}}\right\} .
$$

The actions of $H^{\left(p^{\prime}\right), \infty}$ and of $\Pi^{\infty}$ on $V(H)^{\left(p^{\prime}\right)}$ stabilize $\widetilde{A}_{e, H}$. Let

$$
A_{e, H}=\left(\widetilde{A}_{e, H}^{\left(p^{\prime}\right)} / H^{\left(p^{\prime}\right), \infty}\right) / \Pi^{\infty} .
$$

Thus $A_{e, H}$ is a subset of $V_{\Pi \infty}(H)$. An element $D \subset \Pi$ of $\widetilde{V}(\Pi)_{H}$ is mapped to $A_{e, H}$ by $\widehat{V}(\Pi)_{H} \rightarrow V_{\Pi^{\infty}}$ if and only if the image of $D$ in $\Pi^{\left(p^{\prime}\right)}$ is a representative of $e$. If $Y \rightarrow X$ is the pointed Galois cover corresponding to $H$, then $A_{e, H}$ can be identified with $A_{z, Y}$, as defined in (1) where $z$ is the node of $\mathcal{X}_{Y}$ corresponding to $e$.

The full subgraph $\mathbb{G}\left(A_{e, H}\right)$ of $\mathbb{G}_{\Pi \infty}(H)$ with vertices

$$
A_{e, H} \cup\left\{i_{H, \Pi}\left(D_{1}\right), i_{H, \Pi}\left(D_{2}\right)\right\}
$$

is a line (indeed, the embedding $\left|\mathbb{G}\left(A_{e, H}\right)\right| \subset\left|\mathbb{T}_{\mathcal{X}_{Y}}\right| \subset X^{\infty}$ identifies $\left|\mathbb{G}\left(A_{e, H}\right)\right|$ with $\bar{S}_{z} \simeq[0,1]$ where $z$ is the edge of $\mathbb{T}_{X}$ corresponding to $e$ ), so that $A_{e, H}$ is naturally a totally ordered set for which $D_{1}$ is the minimal element and $D_{2}$ is the maximal 
one. If $\bar{e}=\left[\left(D_{2}, D_{1}\right)\right] \in E(\Pi)^{\infty}$ is the same edge of $\mathbb{G}(\Pi)^{\infty}$ with the opposite orientation, then there is an obvious bijection $A_{e, H} \simeq A_{\bar{e}, H}$, which is decreasing.

Let $H^{\prime} \subset H \subset \Pi$ be two finite index normal subgroups of $\Pi$. The injective map $V_{\Pi \infty}(H) \rightarrow V_{\Pi \infty}\left(H^{\prime}\right)$ maps $A_{e, H}$ to $A_{e, H^{\prime}}$. The induced map $A_{e, H} \rightarrow A_{e, H^{\prime}}$ is increasing. Let

$$
A_{e}:=\underset{H}{\lim } A_{e, H},
$$

where $H$ runs through finite index normal subgroups of $\Pi$. By identifying $A_{e, H}$ with $A_{z, Y}$, one gets an increasing bijection $A_{e} \simeq A_{z}$. Since $X$ satisfies resolution of nonsingularities, $A_{z}$ can be identified with the set of points of type 2 of $S_{z}$. Thus $A_{e}$ is an ordered set which is, noncanonically, isomorphic to $\mathbf{Q} \cap(0,1)$. Let $\widehat{A}_{e}$ be the Dedekind completion of $A_{e} ; \widehat{A}_{e}$ is an ordered topological space noncanonically isomorphic to $[0,1]$. The decreasing bijections $A_{e, H} \rightarrow A_{\bar{e}, H}$ are compatible and therefore induce a homeomorphism $\phi_{e}: \widehat{A}_{e} \rightarrow \widehat{A}_{\bar{e}}$. Let us denote by $0_{e}$ (resp. $1_{e}$ ) the minimal element of $\widehat{A}_{e}$. We then define the topological space

$$
S(\Pi)^{\infty}:=\left(V(\Pi)^{\infty} \coprod_{e \in E(\Pi)^{\infty}} \widehat{A}_{e}\right) / \sim
$$

where $\sim$ is generated by

$$
\forall\left(D, D^{\prime}\right) \in E(\Pi)^{\infty}, D \sim 0_{\left(D, D^{\prime}\right)}, \quad \forall e \in E(\Pi)^{\infty} \forall x \in \widehat{A}_{e}, x \sim \phi_{e}(x) .
$$

Since $\widehat{A}_{e}$ is noncanonically homeomorphic to $[0,1], S(\Pi)^{\infty}$ is noncanonically homeomorphic to the geometric realization of $\mathbb{G}(\Pi)^{\infty}$.

If $H$ is a finite index open normal subgroup of $\Pi$, one similarly gets a topological space $S(H)^{\infty}$ and the action of $\Pi$ on $H$ by conjugacy induces an action of $\Pi$ on $S(H)^{\infty}$.

Let $\tilde{e} \in E(H)^{\left(p^{\prime}\right)}$ and $\tilde{e}_{0} \in E(\Pi)^{\left(p^{\prime}\right)}$ be such that $\iota_{H, \Pi}^{\left(p^{\prime}\right)}\left(D_{\tilde{e}}\right)$ is an open subgroup of $D_{\tilde{e}_{0}}$. Let $H^{\prime} \subset H$ be an open normal subgroup of $\Pi$. Then $\widetilde{A}_{e, H^{\prime}} \subset \widetilde{A}_{e_{0}, H^{\prime}}$ as subsets of $V\left(H^{\prime}\right)^{\left(p^{\prime}\right)}$. This yields a map

$$
A_{e, H^{\prime}}:=\widetilde{A}_{e, H^{\prime}} / H \rightarrow A_{e_{0}, H^{\prime}}:=A_{e_{0}, H^{\prime}} .
$$

Since open normal subgroups of $\Pi$ which are inside $H$ are cofinal among open normal subgroups of $\Pi$ and among normal subgroups of $H$, by taking colimits one gets a map

$$
\alpha_{e, e_{0}}: A_{e} \rightarrow A_{e_{0}} .
$$

Lemma 3.8. There exists at most one continuous map $\psi_{H, \Pi}: S(H)^{\infty} \rightarrow S(\Pi)^{\infty}$ such that:

(i) if $e \in E(H)^{\infty}$ and $\iota_{H, \Pi}^{\left(p^{\prime}\right)}\left(D_{\tilde{e}}\right)=1$, then $\psi_{H, \Pi}$ is constant on $\widehat{A}_{e}$;

(ii) if $\tilde{e} \in E(H)^{\left(p^{\prime}\right)}$ and $\tilde{e}_{0} \in E(\Pi)^{\left(p^{\prime}\right)}$ are such that $\iota_{H, \Pi}^{\left(p^{\prime}\right)}\left(D_{\tilde{e}}\right)$ is an open subgroup of $D_{\tilde{e}_{0}}$, then $\psi_{H, \Pi \mid A_{e}}=\alpha_{e, e_{0}}$. 
Proof. Assume $\psi$ and $\psi^{\prime}$ satisfy the stated conditions. For every $\tilde{e} \in E(H)^{\left(p^{\prime}\right)}$ such that $\iota_{H, \Pi}^{\left(p^{\prime}\right)}\left(D_{\tilde{e}}\right) \neq 1$, by (ii), $\psi=\psi^{\prime}$ on $A_{e}$ and therefore on $\widehat{A}_{e}$. For every $\tilde{v} \in V(H)^{\left(p^{\prime}\right)}$ such that $\iota_{H, \Pi}^{\left(p^{\prime}\right)}\left(D_{\tilde{v}}\right) \neq 1$, there exists $\tilde{e} \in E(H)^{\left(p^{\prime}\right)}$ ending at $\tilde{v}$ such that $\iota_{H, \Pi}^{\left(p^{\prime}\right)}\left(D_{\tilde{v}}\right) \neq 1$, and therefore $\psi(v)=\psi\left(v^{\prime}\right)$. Since $\mathbb{G}(\Pi)^{\infty}$ is connected, one can link every edge and vertex of $\mathbb{G}(\Pi)^{\infty}$ by a finite path to a vertex such that $\iota_{H, \Pi}^{\left(p^{\prime}\right)}\left(D_{\tilde{v}}\right) \neq 1$. Up to reducing the path one can assume that $\iota_{H, \Pi}^{\left(p^{\prime}\right)}\left(D_{\tilde{e}}\right)=1$ for every edge $e$ of the path. By induction on the length of such a path, one gets $\psi=\psi^{\prime}$ using (i).

There is a $\Pi$-equivariant injection $V(\Pi)^{\left(p^{\prime}\right)} \rightarrow X^{\left(p^{\prime}\right)}$ that maps $D$ to the unique $x \in X^{\left(p^{\prime}\right)}$ such that $D=D_{x}$. By quotienting by $\Pi^{\infty}$, one gets a $\Pi$-equivariant injection $V(\Pi)^{\infty} \rightarrow X^{\infty}$. Similarly, for every finite index subgroup $H$ of $\Pi$, there is a $\Pi$-equivariant injection $V(H)^{\infty} \rightarrow Y^{\infty}$, which induces by quotienting by $\Pi^{\infty}$ an injection $V_{\Pi \infty}(H) \rightarrow X^{\infty}$. If $H \subset H^{\prime}$ are two finite index normal subgroups, the following diagram is commutative:

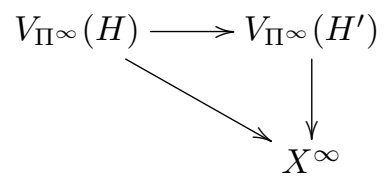

Let $e$ be an edge of $\mathbb{G}(\Pi)^{\infty}$, and let $z$ be the corresponding node of $\mathcal{X}_{k}$. One gets compatible injective maps $A_{e, H} \rightarrow X^{\infty}$, whose images lie in $S_{z}$, hence an injective map $f_{e}: A_{e} \rightarrow S_{z}$, compatible with the reversing of edges. Since $A_{e}$ is dense in $\widehat{A}_{e}$, there is at most one extension of $f_{e}$ to a map $\widehat{A}_{e} \rightarrow X^{\infty}$, necessarily compatible with edges. Since $X$ satisfies resolution of nonsingularities, the image of $A_{e}$ is exactly the set of points of type 2 in $S_{z}$. However, if $\mathcal{X}$ is isomorphic to Spec $O_{K}[X, Y] /(X Y-a)$ in an étale neighborhood of $z$, then $\bar{S}_{z}$ can be identified with $[0, v(a)]$, and the set of points of type 2 of $S_{z}$ is $\mathbf{Q} \cap(0, v(a))$. On $A_{e, H}$ identified with a finite subset of $[0, v(a)]$, the tree structure is simply given by joining the consecutive points. Therefore $A_{e} \rightarrow[0, v(a)]$ is monotone. Hence $f_{e}$ extends to a unique homeomorphism $\tilde{f}_{e}: \widehat{A}_{e} \rightarrow S_{z}$ that preserves the endpoints and is compatible with the reversing of edges. By gluing these maps, one therefore gets a continuous bijection $f^{\infty}: S(\Pi)^{\infty} \rightarrow S\left(X^{\infty}\right)$, which is a homeomorphism since $S(\Pi)^{\infty}$ is locally compact.

Similarly, if $H$ is a finite index open subgroup of $\Pi$, one gets a $\Pi$-equivariant homeomorphism $S(H)^{\infty} \rightarrow S\left(Y^{\infty}\right)$.

The composition $S(H)^{\infty} \simeq S\left(Y^{\infty}\right) \stackrel{r_{X} f^{\infty} \iota_{Y}}{\longrightarrow} S\left(X^{\infty}\right) \simeq S(\Pi)^{\infty}$, where $f^{\infty}$ is the map $Y^{\infty} \rightarrow X^{\infty}$, satisfies the properties of Lemma 3.8. Therefore there exists a unique map $S(H)^{\infty} \simeq S(\Pi)^{\infty}$ with these properties. 
If $H^{\prime} \subset H$ are two finite index open subgroups of $\Pi$, then, since $H$ is also temp-like, there exists a unique map $S\left(H^{\prime}\right)^{\infty} \rightarrow S(H)^{\infty}$ with the properties of Lemma 3.8 and this map is ח-equivariant by uniqueness. If $H^{\prime \prime} \subset H^{\prime}$, then the diagram

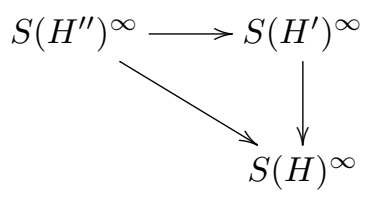

is commutative. One therefore gets a projective system $\left(S(H)^{\infty}\right)$ and one defines

$$
\widetilde{S}(\Pi)={\underset{\leftarrow}{H}}_{\lim } S(H)^{\infty} \text {. }
$$

The equivariant homeomorphisms induce a П-equivariant homeomorphism

$$
S(H)^{\infty} \rightarrow S\left(Y^{\infty}\right)
$$

induce a П-equivariant homeomorphism

$$
\widetilde{S}(\Pi) \rightarrow \lim _{Y} S\left(Y^{\infty}\right)
$$

The maps $|\tilde{X}|_{c} \rightarrow \bar{Y}^{\infty} \stackrel{r_{Y}}{\longrightarrow} S\left(Y^{\infty}\right)$ are compatible and therefore induce a П-equivariant map $|\tilde{X}|_{c} \rightarrow \lim _{Y} S\left(Y^{\infty}\right)$.

Lemma 3.9. The map $|\tilde{X}|_{c} \rightarrow \lim _{Y} S\left(Y^{\infty}\right)$ is a homeomorphism.

Proof. First let us show that the map $r: \bar{X}^{\text {an }}=|\widetilde{X}|_{c} / \Pi \rightarrow \lim _{Y} S\left(Y^{\infty}\right) / \Pi=$ $\lim _{Y} S\left(\mathcal{X}_{Y}\right)$ is a homemorphism (the proof is similar to the proof of Prop. 1.1). Since $r_{\mathcal{X}_{Y}}: \bar{X}^{\text {an }} \rightarrow S\left(\mathcal{X}_{Y}\right)$ is surjective for every $Y$, and $\bar{X}^{\text {an }}$ is compact, $r$ is surjective. Let $x_{1} \neq x_{2} \in \bar{X}^{\text {an }}$; we will show that $r\left(x_{1}\right) \neq r\left(x_{2}\right)$. One can assume $r_{X}\left(x_{1}\right)=r_{X}\left(x_{2}\right)$. Let $\left[x_{1}, x_{2}\right]$ be the smallest subset of $X$ containing $x_{1}$ and $x_{2}$, endowed with the total order such that $x_{1}<x_{2}$. Let $y_{1}<y_{2}$ be two points of type 2 in $\left[x_{1}, x_{2}\right]$ and let $Y \rightarrow X$ be a finite Galois cover such that $y_{1}, y_{2} \in V\left(\mathcal{X}_{Y}\right)$. Then $r_{\mathcal{X}_{Y}}\left(x_{1}\right)<y_{1}<y_{2}<r_{\mathcal{X}_{Y}}\left(x_{2}\right)$ in $\left[x_{1}, x_{2}\right]$, which proves the injectivity of $r_{\mathcal{X}_{Y}}$ and therefore of $r$. Since $\bar{X}^{\text {an }}$ is compact, $r$ is a homeomorphism.

By pulling back along $S\left(X^{\infty}\right) \rightarrow S(X)$, one finds that

$$
r^{\infty}: \bar{X}^{\infty}=\bar{X}^{\text {an }} \times_{S(X)} S\left(X^{\infty}\right) \rightarrow \underbrace{\lim }_{Y} S\left(\mathcal{X}_{Y}\right) \times_{S(X)} S\left(X^{\infty}\right)=\underbrace{\lim }_{Y} S\left(\mathcal{X}_{Y}^{\infty}\right)
$$

is a homeomorphism.

Similarly, the map $\bar{Y}^{\infty} \rightarrow \lim _{Z} S\left(\mathcal{Y}_{Z}^{\infty}\right)$, where $Z$ runs through Galois pointed covers of $Y$ (one can even restrict to $Z$ over $Y$ Galois over $X$ since they are cofinal among Galois pointed covers of $Y$ ), is a homeomorphism for every $Y$ Galois. 
Therefore the map

$$
|\tilde{X}|_{c} \rightarrow \lim _{Z \rightarrow Y \rightarrow X} S\left(\mathcal{Y}_{Z}^{\infty}\right) \rightarrow{\underset{\bigsqcup}{Z}}_{\lim } S\left(Z^{\infty}\right)
$$

is a homeomorphism (the right arrow is a homeomorphism because the full subcategory of the category of morphisms $Y \rightarrow Z$ of pointed Galois covers over $X$ which consists of isomorphisms is a cofinal category).

One thus gets an equivariant homeomorphism

$$
\widetilde{S}(\Pi) \rightarrow|\widetilde{X}|_{c} .
$$

Let $(\bar{X}, D)$ be a $\overline{\mathbf{Q}}_{p}$-marked curve and let $X=\bar{X} \backslash D$. If $Y \rightarrow X$ is a Galois finite étale cover and $\left(\overline{\mathcal{Y}}, \mathcal{D}_{Y}\right)$ is the stable model of $Y$, then $\mathcal{X}_{Y}:=\overline{\mathcal{Y}} / \operatorname{Gal}(Y / X)$ is a semistable model of $\bar{X}$ and one gets a refinement $\phi_{Y}: \mathbb{G}_{Y / X}:=\mathbb{G}_{Y} / \operatorname{Gal}(Y / X)=$

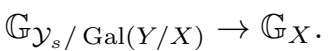

If $X$ satisfies nonresolution of singularities, the family $(\overline{\mathcal{Y}} / \mathrm{Gal}(Y / X))_{Y}$ is cofinal among semistable models of $\bar{X}$. Thus, if $e \in \mathcal{E}(X), A_{e}=\underline{\lim } A_{\phi_{Y}, e}$ and $\bar{X}^{\text {an }} \rightarrow \lim _{Y}\left|\mathbb{G}_{Y / X}\right|_{\text {can }}$ is a homeomorphism.

Theorem 3.10. Let $X_{1}=\bar{X}_{1} \backslash D_{1}$ and $X_{2}=\bar{X}_{2} \backslash D_{2}$ be two marked curves satisfying resolution of nonsingularities and let $\widetilde{X}_{i}$ be a universal pro-tempered cover of $X_{i}$. Let $\psi: \pi_{1}^{\mathrm{temp}}\left(X_{1}, \widetilde{X}_{1}\right) \simeq \pi_{1}^{\mathrm{temp}}\left(X_{2}, \widetilde{X}_{2}\right)$ be an isomorphism. Then there exists a unique homeomorphism $\bar{\psi}:\left|\widetilde{X}_{1}\right|_{c} \rightarrow\left|\widetilde{X}_{2}\right|_{c}$ which is $\pi_{1}^{\text {temp }}$-equivariant in the sense that the following diagram commutes:

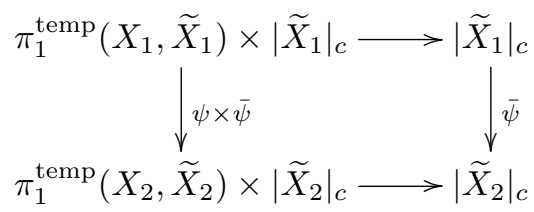

In particular, by quotienting by the tempered fundamental group, one gets a homeomorphism

$$
\bar{X}_{1}^{\text {an }} \rightarrow \bar{X}_{2}^{\text {an }}
$$

Proof. First, assume $\bar{\psi}_{1}, \bar{\psi}_{2}:\left|\widetilde{X}_{1}\right|_{c} \rightarrow\left|\widetilde{X}_{2}\right|_{c}$ are two $\pi_{1}^{\text {temp }}$-equivariant homeomorphisms. Then, if $x \in\left|\widetilde{X}_{1}\right|_{c}$, one has $\psi\left(D_{x}\right)=D_{\bar{\psi}_{1}(x)}=D_{\bar{\psi}_{2}(x)}$. If $x$ is in $\widetilde{V}\left(\widetilde{X}_{1}\right)$, then there exists an open subgroup $H$ of $\pi_{1}^{\text {temp }}\left(X_{1}, \widetilde{X}_{1}\right)$ of finite index such that $\left(D_{x} \cap H\right)^{\left(p^{\prime}\right)}$ is not commutative. Then $\psi(H)$ is a finite index subgroup of $\pi_{1}^{\text {temp }}\left(X_{2}, \widetilde{X}_{2}\right)$, and $\left(D_{\bar{\psi}_{1}(x)} \cap \psi(H)\right)^{\left(p^{\prime}\right)}$ and $\left(D_{\bar{\psi}_{2}(x)} \cap \psi(H)\right)^{\left(p^{\prime}\right)}$ are not commutative. Therefore $\bar{\psi}_{1}(x)$ and $\bar{\psi}_{2}(x)$ are of type 2 and have the same decomposition 
group: according to Proposition $3.5, \bar{\psi}_{1}(x)=\bar{\psi}_{2}(x)$ for every point of $\widetilde{V}\left(\widetilde{X}_{1}\right)$. Since $\widetilde{V}\left(\tilde{X}_{1}\right)$ is dense in $\left|\widetilde{X}_{1}\right|_{c}$, one has $\bar{\psi}_{1}=\bar{\psi}_{2}$.

The morphism $\psi$ induces an equivariant homeomorphism

$$
\widetilde{S}\left(\pi_{1}^{\mathrm{temp}}\left(X_{1}, \widetilde{X}_{1}\right)\right) \rightarrow \widetilde{S}\left(\pi_{1}^{\mathrm{temp}}\left(X_{2}, \widetilde{X}_{2}\right)\right) .
$$

One gets from (3) equivariant homeomorphisms $\left|\widetilde{X}_{1}\right|_{c} \rightarrow \widetilde{S}\left(\pi_{1}^{\text {temp }}\left(X_{1}, \widetilde{X}_{1}\right)\right)$ and $\left|\widetilde{X}_{2}\right|_{c} \rightarrow \widetilde{S}\left(\pi_{1}^{\text {temp }}\left(X_{2}, \widetilde{X}_{2}\right)\right)$. The desired isomorphism is obtained by composition.

Proposition 3.11. Let $\psi_{a}, \psi_{b}: \pi_{1}^{\mathrm{temp}}\left(X_{1}, \widetilde{X}_{1}\right) \rightarrow \pi_{1}^{\mathrm{temp}}\left(X_{2}, \widetilde{X}_{2}\right)$ be two isomorphisms. If $\tilde{\psi}_{a}=\tilde{\psi}_{b}$, then $\psi_{a}=\psi_{b}$.

Proof. Let $g \in \pi_{1}^{\text {temp }}\left(X_{1}, \widetilde{X}_{1}\right)$. If $x_{1} \in\left|\widetilde{X}_{1}\right|$, then

$$
\begin{aligned}
\psi_{a}(g) D_{\tilde{\psi}_{a}\left(x_{1}\right)} \psi_{a}(g)^{-1} & =D_{\tilde{\psi}_{a}\left(g x_{1}\right)}=D_{\tilde{\psi}_{b}\left(x_{1}\right)}=\psi_{b}(g) D_{\tilde{\psi}_{b}\left(x_{1}\right)} \psi_{b}(g)^{-1} \\
& =\psi_{b}(g) D_{\tilde{\psi}_{a}\left(x_{1}\right)} \psi_{b}(g)^{-1}
\end{aligned}
$$

which implies that $g_{0}:=\psi_{a}(g)^{-1} \psi_{b}(g)$ is in the normalizer $N_{\psi_{a}\left(x_{1}\right)}$ of $D_{\psi_{a}\left(x_{1}\right)}$. Since $\psi_{a}$ is bijective, $g_{0} \in \bigcap_{x_{2} \in \widetilde{X}_{2}} N_{x_{2}}$. If $x_{2} \in \widetilde{V}\left(\widetilde{X}_{2}\right)$, then $N_{x_{2}}=D_{x_{2}}$. Therefore $g_{0}\left(x_{2}\right)=x_{2}$ for every $x_{2} \in \widetilde{V}\left(\widetilde{X}_{2}\right)$. Since $X_{2}$ satisfies resolution of nonsingularities, $\widetilde{V}\left(\widetilde{X}_{2}\right)$ is dense in $\widetilde{X}_{2}$, and thus $g_{0}(x)=x$ for every $x \in \widetilde{X}_{2}$. If $x$ is of type 1 , then $D_{x}=\{1\}$. Therefore $g_{0}=1$, i.e. $\psi_{a}(g)=\psi_{b}(g)$.

In particular, if $(X, \tilde{X})$ is a pointed curve satisfying resolution of nonsingularities, one has an injective morphism of groups Aut $\pi_{1}^{\text {temp }}(X, \widetilde{X}) \rightarrow$ Aut $|\widetilde{X}|$.

\section{$\S 4$. Tate curves}

Let $q_{1}, q_{2} \in \overline{\mathbf{Q}}_{p}$ be such that $\left|q_{1}\right|<1$ and $\left|q_{2}\right|<1$. Let $E_{i}=\mathbf{G}_{\mathrm{m}} / q_{i}^{\mathbf{Z}}$ and let $X_{i}=E_{i} \backslash\{1\}$.

If there exists $\sigma \in G_{\mathbf{Q}_{p}}$ such that $q_{1}=\sigma\left(q_{2}\right)$, there is a $\mathbf{C}_{p}$-isomorphism $X_{1} \simeq X_{2} \otimes \mathbf{C}_{p} \mathbf{C}_{p}$ where the base change $\mathbf{C}_{p} \rightarrow \mathbf{C}_{p}$ is $\sigma$. Therefore $X_{1}$ and $X_{2}$ are isomorphic analytic spaces over $\mathbf{Q}_{p}$ and therefore have isomorphic tempered fundamental groups. The following theorem states that the converse is also true:

Theorem 4.1. Let $\psi: \pi_{1}^{\text {temp }}\left(X_{1}, \widetilde{X}_{1}\right) \simeq \pi_{1}^{\text {temp }}\left(X_{2}, \widetilde{X}_{2}\right)$ be an isomorphism. There exists $\sigma \in G_{\mathbf{Q}_{p}}$ such that $q_{1}=\sigma\left(q_{2}\right)$, i.e. $E_{1}$ and $E_{2}$ are isomorphic analytic spaces over $\mathbf{Q}_{p}$.

Remark. The curves $X_{1}$ and $X_{2}$ satisfy the assumptions of Theorem 3.10, and thus $\psi$ induces a homeomorphism $E_{1}^{\text {an }} \rightarrow E_{2}^{\text {an }}$. However, the author does not know, 
even in this situation, if this homeomorphism comes from an analytic morphism. The author does not know how to associate to $\psi$ a particular $\sigma$.

Proof of Theorem 4.1. Recall that $\left|q_{1}\right|=\left|q_{2}\right|$ and that $\psi$ induces a unique equivariant homeomorphism $\tilde{\psi}:\left|\widetilde{X}_{1}\right|_{c} \simeq\left|\widetilde{X}_{2}\right|_{c}$. The induced homeomorphism $E_{1} \simeq E_{2}$ maps $X_{1}$ onto $X_{2}$. Let $\Omega_{i}$ be the universal $\tilde{X}_{i}$-pointed topological cover of $X_{i}$, and $\bar{\psi}:\left|\bar{\Omega}_{1}\right| \rightarrow\left|\bar{\Omega}_{2}\right|$ the homeomorphism induced by $\tilde{\psi}$.

Let $E_{i, l}$ be the unique $\widetilde{X}_{i}$-pointed connected topological cover of $E_{i}$ of degree $l$. Let $X_{i, l}=X_{i} \times_{E_{i}} E_{i, l}$. The isomorphism $\psi$ induces an isomorphism $\psi_{l}: \pi_{1}^{\text {temp }}\left(X_{1, l}, \widetilde{X}_{1}\right) \rightarrow \pi_{1}^{\text {temp }}\left(X_{2, l}, \widetilde{X}_{2}\right)$, whence an isomorphism

$$
\psi_{l, n}: H^{1}\left(X_{2, l}, \mu_{n}\right) \rightarrow H^{1}\left(X_{1, l}, \mu_{n}\right)
$$

functorial in $l$ for divisibility.

Let $\mathbb{G}_{i}$ be the semigraph of $X_{i}$ and let $\mathbb{T}_{i}$ be its universal cover. Let $g$ be the isomorphism $\mathbb{T}_{1} \rightarrow \mathbb{T}_{2}$ induced by $\psi$. One identifies $\bar{\Omega}_{i}$ with $\mathbf{G}_{\mathrm{m}}$ in such a way that $\bar{\psi}(1)=1$ and $\bar{\psi}\left(q_{1}\right)=q_{2}$. If $j \in \mathbf{Z}$, we denote by $e_{i, j}$ the cuspidal edge of $\mathbb{T}_{i}$ corresponding to $q_{i}^{j} \in \bar{\Omega}_{i} \backslash \Omega_{i}$ and by $v_{i, j}$ the vertex of $\mathbb{T}_{i}$ at which $e_{i, j}$ ends. Since $\bar{\psi}(1)=1$ and $\bar{\psi}\left(q_{1}\right)=q_{2}$, one has $g\left(e_{1, j}\right)=e_{2, j}$ and $g\left(v_{1, j}\right)=v_{2, j}$. We denote by $e_{i, j}^{\prime}$ the unique oriented edge joining $v_{i, j}$ to $v_{i, j+1}$.

All the cohomology groups will be cohomology groups for étale cohomology in the sense of algebraic geometry or in the sense of Berkovich (one can replace étale cohomology of $X^{\text {an }}$ by étale cohomology of $X$ thanks to [4, Thm. 3.1]). Since $\Gamma_{l} \simeq \mathbf{Z}$, one has $H^{2}\left(\Gamma, \mu_{n}\right)=0$. Therefore the spectral sequence

$$
H^{p}\left(\Gamma_{l}, H^{q}\left(\Omega, \mu_{n}\right)\right) \Rightarrow H^{n}\left(X_{i, l}, \mu_{n}\right)
$$

of the Galois étale cover $\Omega_{i} \rightarrow X_{i, l}$ gives us an exact sequence of cohomology groups for Berkovich étale topology:

$$
1 \rightarrow \operatorname{Hom}\left(\Gamma_{l}, \mu_{n}\right) \rightarrow H^{1}\left(X_{i, l}, \mu_{n}\right) \rightarrow H^{1}\left(\Omega_{i}, \mu_{n}\right)^{\Gamma_{l}} \rightarrow 1 .
$$

The map $O^{*}\left(\Omega_{i}\right) / O^{*}\left(\Omega_{i}^{n}\right) \rightarrow H^{1}\left(\Omega_{i}, \mu_{n}\right)$ given by Kummer theory (see [3, Prop. 4.1.7] for the Kummer exact sequence in Berkovich étale topology) induces a morphism

$$
\left(O^{*}\left(\Omega_{i}\right) / O^{*}\left(\Omega_{i}\right)^{n}\right)^{\Gamma_{l}} \rightarrow H^{1}\left(\Omega_{i}, \mu_{n}\right)^{\Gamma_{l}},
$$

which turns out to be an isomorphism (cf. [8, §1.4.2]); hence an exact sequence

$$
1 \rightarrow \operatorname{Hom}\left(\Gamma_{l}, \mu_{n}\right) \rightarrow H^{1}\left(X_{i, l}, \mu_{n}\right) \rightarrow\left(O^{*}\left(\Omega_{i}\right) / O^{*}\left(\Omega_{i}\right)^{n}\right)^{\Gamma_{l}} \rightarrow 1 .
$$

where $\Gamma_{l}=\operatorname{Gal}\left(\Omega_{i} / X_{i, l}\right)$.

One can describe $O^{*}\left(\Omega_{i}\right)$ in terms of currents (as done in [14] for Mumford curves). If $A$ is a ring, an $A$-current on $\mathbb{T}_{i}$ is a function $c:\left\{e_{i, j}\right\}_{j \in \mathbf{Z}} \amalg\left\{e_{i, j}^{\prime}\right\}_{j \in \mathbf{Z}} \rightarrow A$ 
such that for every $j \in \mathbf{Z}, c\left(e_{i, j+1}^{\prime}\right)=c\left(e_{i, j}^{\prime}\right)+c\left(e_{i, j+1}\right)$. Let $C\left(\mathbb{T}_{i}, A\right)$ be the $A$ module of $A$-currents on $\mathbb{T}_{i}$. There is a natural isomorphism $\alpha_{i}: C\left(\mathbb{T}_{i}, \mathbf{Z}\right) \rightarrow$ $O^{*}\left(\Omega_{i}\right) / \mathbf{C}_{p}^{*}$ defined by

$$
\alpha_{i}(c)=x^{c\left(e_{i, 0}^{\prime}\right)} \prod_{j \geq 1}\left(\frac{x-q_{i}^{j}}{x}\right)^{c\left(e_{i, j}\right)} \prod_{j \leq 0}\left(\frac{x-q_{i}^{j}}{q_{i}^{j}}\right)^{c\left(e_{i, j}\right)} .
$$

Conversely, if $f \in O^{*}\left(\Omega_{i}\right)$, one can compute $\alpha_{i}^{-1}(f)$ in the following way. For every $j \in \mathbf{Z}$, the restriction of $f$ to the open annulus $U_{j}=\left\{\left.z \in \mathbf{P}^{1, \text { an }}|| q_{i}^{j}|<| x\right|_{z}<\right.$ $\left.\left|q_{i}^{j+1}\right|\right\}$ can be written in a unique way as $f(x)=x^{m_{j}} g_{j}(x)$ where $m_{j} \in \mathbf{Z}$ and $\left|g_{j}\right|$ is constant on $U_{j}$. One has $\alpha_{i}^{-1}(f)\left(e_{i, j}^{\prime}\right)=m_{j}$. Similarly, the restriction of $f$ to the punctured open disk $V_{j}=\left\{z \in \mathbf{P}^{1 \text {,an }}|0<| x-\left.q_{i}^{j}\right|_{z}<\left|q_{i}^{j}\right|\right\}$ can be written in a unique way as $f(x)=x^{n_{j}} f_{j}(x)$ where $n_{j} \in \mathbf{Z}$ and $\left|f_{j}\right|$ is constant on $V_{j}$. One has $\alpha_{i}^{-1}(f)\left(e_{i, j}\right)=n_{j}$.

Therefore, one gets an isomorphism $\alpha_{i, n}: O^{*}\left(\Omega_{i}\right) / O^{*}\left(\Omega_{i}\right)^{n} \rightarrow C\left(\mathbb{T}_{i}, \mathbf{Z} / n \mathbf{Z}\right)$, hence an exact sequence

$$
1 \rightarrow \operatorname{Hom}\left(\Gamma_{l}, \mu_{n}\right) \rightarrow H^{1}\left(X_{i, l}, \mu_{n}\right) \rightarrow C\left(\mathbb{T}_{i}, \mathbf{Z} / n \mathbf{Z}\right)^{\Gamma_{l}} \rightarrow 1
$$

Since $\lim _{l} \operatorname{Hom}\left(\Gamma_{l}, \mu_{n}\right)=0$, it induces an isomorphism

$$
a_{i}: \underset{l}{\lim } H^{1}\left(X_{i, l}, \mu_{n}\right) \rightarrow C\left(\mathbb{T}_{i}, \mathbf{Z} / n \mathbf{Z}\right)^{(\Gamma)},
$$

where $C\left(\mathbb{T}_{i}, \mathbb{Z} / n \mathbb{Z}\right)^{(\Gamma)}$ is the set of $\mathbb{Z} / n \mathbb{Z}$-currents on $\mathbb{T}_{i}$ that are invariant under some finite index subgroup of $\Gamma$.

Consider the diagram

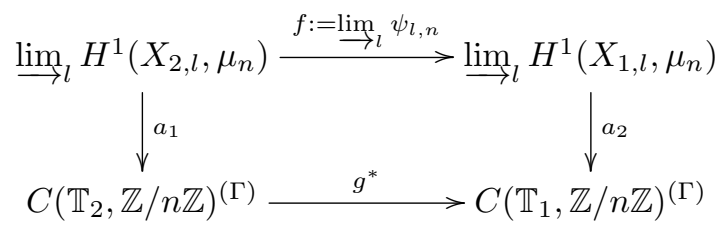

where the lower arrow is induced by $g: \mathbb{T}_{1} \rightarrow \mathbb{T}_{2}$.

The following lemma shows that this diagram is commutative up to a constant in $(\mathbf{Z} / n \mathbf{Z})^{*}$ (cf. [8, Prop. 13] for a similar result for Mumford curves of genus greater than 2).

Proposition 4.2. There exists a unique $\alpha \in(\mathbf{Z} / n \mathbf{Z})^{*}$ such that $a_{2} f=\alpha g^{*} a_{1}$.

Proof. Let $\tilde{f}=a_{2} f a_{1}^{-1}$. We have to show that there exists $\lambda \in(\mathbf{Z} / n \mathbf{Z})^{*}$ such that for every $c \in C\left(\mathbb{T}_{2}, \mathbb{Z} / n \mathbb{Z}\right)^{(\Gamma)}$ and every edge $e$ of $\mathbb{T}_{1}, \tilde{f}(c)(e)=\lambda c(g(e))$. 
Let $j \in \mathbf{Z}$. According to [9, Lem. 4.2], a finite cover of $X_{1, l}$ is ramified at $e_{1, j}$ if and only if the corresponding cover of $X_{2, l}$ is unramified at $e_{2, j}$. Therefore $\tilde{f}(c)\left(e_{1, j}\right)=0$ if and only if $c\left(e_{2, j}\right)=0$. Therefore there exists $\lambda_{j} \in(\mathbf{Z} / n \mathbf{Z})^{*}$ such that $\tilde{f}(c)\left(e_{1, j}\right)=\lambda_{j} c\left(e_{2, j}\right)$ for every $c \in C\left(\mathbb{T}_{2}, \mathbb{Z} / n \mathbb{Z}\right)^{(\Gamma)}$. Let $l$ be a positive integer. Let $j \in \mathbf{Z}$ and let $c_{j}$ be the $\Gamma_{l}$-invariant current defined by

$$
\begin{aligned}
& c_{j}\left(e_{1, k}\right)= \begin{cases}1 & \text { if } k=j \bmod l, \\
-1 & \text { if } k=j+1 \bmod l, \\
0 & \text { otherwise, }\end{cases} \\
& c_{j}\left(e_{1, k}^{\prime}\right)= \begin{cases}0 & \text { if } k \in[j+1, j+l-1] \bmod l, \\
1 & \text { if } k=j \bmod l .\end{cases}
\end{aligned}
$$

If $l$ is large enough (for example if $l \geq 2+2\left(v_{p}(n)+2\right) /\left|q_{1}\right|$ according to [9, Cor. $4.10])$, the $\mu_{n}$-torsor corresponding to $c_{j}$ is split at $v_{1, j+\lceil(l+1) / 2\rceil}$. Therefore, the $\mu_{n}$-torsor corresponding to $\tilde{f}\left(c_{e}\right)$ is split at $v_{2, j+\lceil(l+1) / 2\rceil}$, which implies, according to [9, Prop. 4.11], that $\tilde{f}\left(c_{j}\right)$ is zero at all the edges ending at $v_{2, j+\lceil(l+1) / 2\rceil}$. Then $\tilde{f}\left(c_{j}\right)\left(e_{2, j}\right)=\lambda_{j}, \tilde{f}\left(c_{j}\right)\left(e_{2, j+1}\right)=\lambda_{j+1}, \tilde{f}\left(c_{j}\right)\left(e_{2, k}\right)=0$ for all $k \neq j, j+1 \bmod l$, and is zero for some noncuspidal edge between $v_{2, j+1}$ and $v_{2, j+l}$. Therefore $\tilde{f}\left(c_{j}\right)=$ $\lambda_{j} g^{*}\left(c_{j}\right)$ and $\lambda_{j}=\lambda_{j+1}$. Thus $\lambda_{j}$ does not depend on $j$, and we simply denote it by $\lambda$. The group of $\Gamma_{l}$-equivariant currents is generated by $\left(c_{j}\right)_{j \in[0,2 l-1]}$ so that $\tilde{f}(c)=\lambda g^{*}(c)$ for every current $c$, which ends the proof.

Let $A_{i}$ be the multiplicative group of nonzero meromorphic functions on $\mathbf{G}_{\mathrm{m}}$ with no poles and no zeroes on $\Omega_{i} \subset \mathbf{G}_{\mathrm{m}}$. Let $B_{i} \subset A_{i}$ be the subgroup of $A_{i}$ consisting of all functions for which 1 is neither a pole nor a zero. Let $A_{i}^{\prime} \subset \Omega^{1}\left(\Omega_{i}\right)$ be the group of regular differentials on $\mathbf{G}_{\mathrm{m}}$ with no poles on $\Omega_{i}$.

The map $d \log \circ \alpha_{i}: C\left(\mathbb{T}_{i}, \mathbf{Z}\right) \rightarrow A_{i}^{\prime}$ can be extended by linearity to a map $\delta_{i}: C\left(\mathbb{T}_{i}, \mathbf{Z}_{p}\right) \rightarrow A_{i}^{\prime}$ defined by

$$
\delta_{i}(c)=c\left(e_{i, 0}^{\prime}\right) \frac{d x}{x}+\sum_{j \geq 1} c\left(e_{i, j}\right)\left(\frac{d x}{x-q_{i}^{j}}-\frac{d x}{x}\right)+\sum_{j \leq 0} c\left(e_{i, j}\right) \frac{d x}{x-q_{i}^{j}} .
$$

If $z \in \mathbf{G}_{\mathrm{m}}\left(\mathbf{C}_{p}\right)$ and $\omega \in A_{i}^{\prime}$, we denote by $\operatorname{ord}_{z}(\omega) \in \mathbf{Z}_{\geq-1}$ the (x-z)-adic valuation of $\frac{\omega}{d x} \in \frac{1}{x-z} \mathbf{C}_{p}[[x-z]]$.

Lemma 4.3. Let $c$ be in $C\left(\mathbb{T}_{1}, \mathbf{Z}_{p}\right)$, and let $z \in \mathbf{G}_{\mathrm{m}}\left(\mathbf{C}_{p}\right)$. Assume that $\bar{\psi}(z)$ is also of type 1 , i.e. $\bar{\psi}(z) \in \mathbf{G}_{\mathrm{m}}\left(\mathbf{C}_{p}\right)$. Then $\operatorname{ord}_{z}\left(\delta_{1}(c)\right)=\operatorname{ord}_{\bar{\psi}(z)}\left(\delta_{2}\left(g^{*}(c)\right)\right)$.

Proof. Let $J=\left\{j \in \mathbf{Z} \mid C\left(e_{1, j}\right) \neq 0\right\}$. For $z=q_{1}^{j}$ with $j \in J$, one has $\bar{\psi}\left(q_{1}^{j}\right)=q_{2}^{j}$ and thus $\operatorname{ord}_{q_{1}^{j}}\left(\delta_{1}(c)\right)=\operatorname{ord}_{q_{2}^{j}}\left(\delta_{2}\left(g^{*}(c)\right)\right)=-1$. One can thus assume that $z \in$ $\mathbf{G}_{\mathrm{m}}\left(\mathbf{C}_{p}\right) \backslash\left\{q_{1}^{j}\right\}_{j \in J}$ and therefore $\operatorname{ord}_{z}\left(\delta_{1}(c)\right), \operatorname{ord}_{\bar{\psi}(z)}\left(\delta_{2}\left(g^{*}(c)\right)\right) \geq 0$. 
Let $c_{n} \in C\left(\mathbb{T}_{1}, \mathbf{Z}\right)^{(\Gamma)}$ be such that $\left|c_{n}\left(e_{1, j}\right)-c\left(e_{1, j}\right)\right|,\left|c_{n}\left(e_{1, j}^{\prime}\right)-c\left(e_{1, j}^{\prime}\right)\right| \leq p^{-n}$ if $-|j|_{\infty} \log _{p}\left|q_{1}\right| \leq n+p /(p-1)$.

Then $\delta_{1}\left(c_{n}\right) \rightarrow \delta_{1}(c)$ on every affinoid subspace of $\mathbf{G}_{\mathrm{m}} \backslash\left\{q_{1}^{j}\right\}_{j \in J}$. Similarly $\delta_{2}\left(g^{*}\left(c_{n}\right)\right) \rightarrow \delta_{2}\left(g^{*}(c)\right)$ on every affinoid subspace of $\mathbf{G}_{\mathrm{m}} \backslash\left\{q_{2}^{j}\right\}_{j \in J}$. Therefore

$$
\operatorname{ord}_{z}\left(\delta_{1}(c)\right) \geq m \Leftrightarrow \forall \epsilon>0 \exists N \forall n \geq N, \sum_{z^{\prime} \in D(z, \epsilon)} \operatorname{ord}_{z^{\prime}}\left(\delta_{1}\left(c_{n}\right)\right) \geq m .
$$

Therefore it is enough to prove the lemma for every $c_{n}$. Assume $c \in C\left(\mathbb{T}_{1}, \mathbf{Z}\right)^{(\Gamma)}$. Let $N$ be such that $c \in C\left(\mathbb{T}_{1}, \mathbf{Z}\right)^{\Gamma_{N}}$. The isomorphism $\psi$ induces an isomorphism $\psi_{N}: \pi_{1}^{\text {temp }}\left(X_{1, N}\right) \rightarrow \pi_{1}^{\text {temp }}\left(X_{2, N}\right)$.

Consider the image $Y_{1}$ of $c$ under the map

$$
C\left(\mathbb{T}_{1}, \mathbf{Z}\right) \rightarrow O^{*}\left(\Omega_{1}\right) \rightarrow H^{1}\left(\Omega_{1}, \mathbf{Z}_{p}(1)\right),
$$

and let $Y_{1, n}$ be the induced $\mu_{p^{n}}$-torsor on $\Omega_{1}$; it extends to an unramified $\mu_{p^{n}}$ torsor of $\Omega_{1} \backslash\left\{q_{1}^{j}\right\}_{j \in J}$. Let $z_{1, n}$ be the point of $\Omega_{1}$ such that $Y_{1, n}$ is not split at $z_{1, n}$ but is split on $\left[z, z_{1, n}\right)$. Consider the image $Y_{2}$ of $g^{*}(c)$ under the map $C\left(\mathbb{T}_{2}, \mathbf{Z}\right) \rightarrow$ $O^{*}\left(\Omega_{2}\right) \rightarrow H^{1}\left(\Omega_{2}, \mathbf{Z}_{p}(1)\right)$, let $Y_{2, n}$ be the induced $\mu_{p^{n}}$-torsor on $\Omega_{2}$, and let $z_{2, n}$ be the point of $\Omega_{2}$ such that $Y_{2, n}$ is not split at $z_{2, n}$ but is split on $\left[\bar{\psi}(z), z_{2, n}\right)$. Since $c$ is $\Gamma_{N}$-invariant, $Y_{i, n}$ is in the image of $\operatorname{Hom}\left(\pi_{1}^{\text {temp }}\left(X_{i, N}\right), \mu_{p^{n}}\right)=H^{1}\left(X_{i, N}, \mu_{p^{n}}\right) \rightarrow$ $H^{1}\left(\Omega_{i}, \mu_{p^{n}}\right)$. For every preimage $\beta_{i}$ of $Y_{i, n}$ in $\operatorname{Hom}\left(\pi_{1}^{\text {temp }}\left(X_{i, N}\right), \mu_{p^{n}}\right), Y_{i, n}$ is split at a point $z^{\prime} \in \mathbf{G}_{\mathrm{m}}$ if and only if $D_{z^{\prime}} \subset \operatorname{Ker} \beta_{i}$, where $D_{z^{\prime}}$ is a decomposition group of $z^{\prime}$ in $\pi_{1}^{\text {temp }}\left(X_{i, N}\right)$. According to Proposition 4.2 , there exists $\alpha \in\left(\mathbf{Z} / p^{n} \mathbf{Z}\right)^{*}$ such that, if $\beta_{1}$ is a preimage of $Y_{1, n}$ in $\operatorname{Hom}\left(\pi_{1}^{\text {temp }}\left(X_{1, N}\right), \mu_{p^{n}}\right)$, then $\alpha \beta_{1} \psi_{N}^{-1}$ is a preimage of $Y_{2, n}$ in $\operatorname{Hom}\left(\pi_{1}^{\text {temp }}\left(X_{2, N}\right), \mu_{p^{n}}\right)$. Since $\operatorname{Ker}\left(\alpha \beta_{1} \psi_{N}^{-1}\right)=\psi\left(\operatorname{Ker} \beta_{1}\right)$ and $D_{\bar{\psi}\left(z^{\prime}\right)}=\psi_{N}\left(D_{z^{\prime}}\right)$, one finds that $Y_{1, N}$ is split at $z^{\prime}$ if and only if $Y_{2, N}$ is split at $\bar{\psi}\left(z^{\prime}\right)$, and therefore $z_{2, n}=\bar{\psi}\left(z_{1, n}\right)$.

Let $c_{0} \in C\left(\mathbb{T}_{1}, \mathbf{Z}\right)^{\Gamma}$ be defined by $c_{0}\left(e_{1, i}\right)=0$ and $c_{0}\left(e_{1, i}^{\prime}\right)=1$ for every $i \in \mathbf{Z}$. Then $\alpha_{1}\left(c_{0}\right)(x)=x$ and $\alpha_{2}\left(g^{*} c_{0}\right)(x)=x$. Let $\phi_{1, n}\left(\right.$ resp. $\left.\phi_{2, n}\right)$ be a preimage of $c_{0} \bmod p^{n}\left(\operatorname{resp} . g^{*} c_{0} \bmod p^{n}\right)$ by the map $\operatorname{Hom}\left(\pi_{1}^{\text {temp }}\left(X_{1}, \widetilde{X}_{1}\right), \mu_{p^{n}}\right)=$ $H^{1}\left(X_{1}, \mu_{p^{n}}\right) \rightarrow C\left(\mathbb{T}_{1}, \mu_{p^{n}}\right)^{\Gamma}\left(\right.$ resp. $\operatorname{Hom}\left(\pi_{1}^{\text {temp }}\left(X_{2}, \widetilde{X}_{2}\right), \mu_{p^{n}}\right)=H^{1}\left(X_{2}, \mu_{p^{n}}\right) \rightarrow$ $\left.C\left(\mathbb{T}_{2}, \mu_{p^{n}}\right)^{\Gamma}\right)$. Let $z_{1, n}^{\prime}=b_{z,|z| p^{-n-1 /(p-1)}} \in \mathbf{G}_{\mathrm{m}}$ and $z_{2, n}^{\prime}=b_{\bar{\psi}(z),|\bar{\psi}(z)| p^{-n-\frac{1}{p-1}}} \in \mathbf{G}_{\mathrm{m}}$. According to [9, Lem. 4.2], $z_{1, n}^{\prime}$ is characterized in $\mathbf{G}_{\mathrm{m}}$ by the fact that $c_{\mathrm{can}, p^{n}}$ is not split at $z_{1, n}^{\prime}$ but is split above $\left[z, z_{1, n}^{\prime}\right)$. Therefore $z_{1, n}^{\prime}$ is also characterized by the

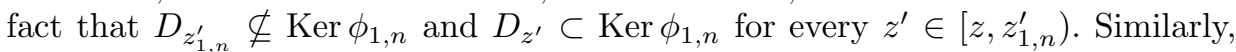
$z_{2, n}^{\prime}$ is characterized by the fact that $D_{z_{2, n}^{\prime}} \nsubseteq \operatorname{Ker} \phi_{2, n}$ and $D_{z^{\prime}} \subset \operatorname{Ker} \phi_{2, n}$ for every $z^{\prime} \in\left[z, z_{2, n}^{\prime}\right)$. According to 4.2 , one can choose $\phi_{2, n}$ to be $\alpha \phi_{1, n} \psi^{-1}$, so that $\operatorname{Ker} \phi_{2, n}=\psi\left(\operatorname{Ker} \phi_{1, n}\right)$. Therefore, since $\bar{\psi}$ is compatible with decomposition groups, $z_{2, n}^{\prime}=\bar{\psi}\left(z_{1, n}^{\prime}\right)$. 
Using Proposition 2.4, one gets

$$
\begin{aligned}
\operatorname{ord}_{z}\left(\delta_{1}(c)\right)+1 & =\lim _{n} \frac{1}{n} \inf \left\{m \mid z_{1, m}^{\prime} \in\left[z, z_{1, n}\right]\right\} \\
& =\lim _{n} \frac{1}{n} \inf \left\{m \mid \bar{\psi}\left(z_{1, m}^{\prime}\right) \in\left[\bar{\psi}(z), \bar{\psi}\left(z_{1, n}\right)\right]\right\} \\
& =\lim _{n} \frac{1}{n} \inf \left\{m \mid z_{2, m}^{\prime} \in\left[\bar{\psi}(z), z_{2, n}\right]\right\}=\operatorname{ord}_{\bar{\psi}(z)}\left(\delta_{2}\left(g^{*} c\right)\right)+1 .
\end{aligned}
$$

Let $\mu: \mathbf{N}_{>0} \rightarrow\{-1,0,1\}$ be the Möbius function. Let $n \geq 1$. Let $c_{n} \in$ $C\left(\mathbb{T}_{1}, \mathbf{Z}\right)$ be defined by

- $c_{n}\left(e_{1, j}\right)=0$ if $j \leq 0$;

- $c_{n}\left(e_{1, j}^{\prime}\right)=0$ if $j \leq 0$;

- $c_{n}\left(e_{1, j}\right)=\mu(j / n)$ if $j \geq 1$ and $j=0 \bmod n$;

- $c_{n}\left(e_{1, j}\right)=0$ if $j \geq 1$ and $j \neq 0 \bmod n$;

- $c_{n}\left(e_{1, j}^{\prime}\right)=\sum_{k=1}^{\lfloor j / n\rfloor} \mu(k)$ if $j \geq 1$.

The associated differentials are:

$$
\delta_{1}\left(c_{n}\right)=\sum_{j \geq 1} \mu(j)\left(\frac{1}{x-q_{1}^{n j}}-1\right) d x, \quad \delta_{2}\left(g^{*}\left(c_{n}\right)\right)=\sum_{j \geq 1} \mu(j)\left(\frac{1}{x-q_{2}^{n j}}-1\right) d x .
$$

By evaluating $\delta_{1}\left(c_{n}\right)$ and $\delta_{2}\left(g^{*}\left(c_{n}\right)\right)$ at 1 in $\mathbf{C}_{p} d x$, one gets

$$
\delta_{1}\left(c_{n}\right)(1)=\sum_{j \geq 1} \mu(j) \frac{q_{1}^{j n}}{1-q_{1}^{j n}} d x=\sum_{j \geq 1} \sum_{k \geq 1} \mu(j) q_{1}^{k j n} d x=\sum_{d \geq 1} \sum_{j \mid d} \mu(j) q_{1}^{d n} d x=q_{1}^{n} d x,
$$

and similarly $\delta_{2}\left(g^{*}\left(c_{n}\right)\right)(1)=q_{2}^{n} d x$. Let $c_{0}$ be defined by $c_{0}\left(e_{1, j}\right)=0$ and $c_{0}\left(e_{1, j}\right)=1$. Then $\delta_{1}\left(c_{0}\right)=\delta_{2}\left(g^{*}\left(c_{0}\right)\right)=\frac{d x}{x}$ and $\delta_{1}\left(c_{0}\right)(1)=\delta_{2}\left(g^{*}\left(c_{0}\right)\right)(1)=d x$.

If $P=\sum_{n \geq 0} a_{n} X^{n} \in \mathbf{Z}_{p}[X]$, let $c_{P}=\sum_{n \neq 0} a_{n} c_{n} \in C\left(\mathbb{T}_{1}, \mathbf{Z}_{p}\right)$, so that $\delta_{1}\left(c_{P}\right)(1)=P\left(q_{1}\right) d x$ and $\delta_{2}\left(g^{*}\left(c_{P}\right)\right)(1)=P\left(q_{2}\right) d x$.

According to Lemma $4.3, \delta_{1}\left(c_{P}\right)(1)=0$ if and only if $\delta_{2}\left(g^{*}\left(c_{P}\right)\right)(1)=0$. Therefore $P\left(q_{1}\right)=0$ if and only if $P\left(q_{2}\right)=0$ for every $P \in \mathbf{Z}_{p}[X]$, which implies the result.

\section{References}

[1] Y. André, Period mappings and differential equations: From $\mathbf{C}$ to $\mathbf{C}_{p}$, MSJ Memoirs 12, Math. Soc. of Japan, Tokyo, 2003. Zbl 1029.14006 MR 1978691

[2] V. G. Berkovich, Spectral theory and analytic geometry over non-archimedian fields, Math. Surveys Monogr. 33, Amer. Math. Soc., Providence, 1990. Zbl 0715.14013 MR 1070709

[3] Étale cohomology for non-archimedean analytic spaces, Publ. Math. Inst. Hautes Études Sci. 78 (1993), 5-161. Zbl 0804.32019 MR 1259429 
[4] V. G. Berkovich, On the comparison theorem for étale cohomology of non-Archimedean analytic spaces, Israel J. Math. 92 (1995), 45-59. Zbl 0864.14011 MR 1357745

[5] A. J. de Jong, Étale fundamental group of non-archimedean analytic spaces, Compos. Math. 97 (1995), 89-118. Zbl 0864.14012 MR 1355119

[6] A. G. B. Lauder and D. Q. Wan, Computing zeta functions of Artin-Schreier curves over finite fields, London Math. Soc. J. Comput. Math. 5 (2002), 34-55. Zbl 1067.11078 MR 1916921

[7] E. Lepage, Tempered fundamental group, in Séminaires \& Congrès, to appear.

[8] _ . Tempered fundamental group and graph of the stable reduction, in The arithmetic of fundamental group - PIA 2010, Contrib. Math. Comput. Sci. 2, to appear.

[9] Kyoto Univ. 46 (2010), 849-897. Zbl 1213.14047 MR 2791009

[10] Q. Liu, Stable reduction of finite covers of curves, Compos. Math. 142 (2006), 101-118. Zbl 1108.14020 MR 2196764

[11] S. Mochizuki, Semi-graphs of anabelioids, Publ. RIMS Kyoto Univ. 42 (2006), 221-322. Zbl 1113.14025 MR 2215441

[12] F. Pop and J. Stix, Arithmetic in the fundamental group of a $p$-adic curve, submitted.

[13] A. Tamagawa, Resolution of nonsingularities of families of curves, Publ. RIMS Kyoto Univ. 40 (2004), 1291-1336. Zbl 1078.14037 MR 2105709

[14] M. van der Put, Les fonctions thêta d'une courbe de Mumford, in Groupe d'étude d'analyse ultramétrique, 9e année: 1981/82, no. 1, exp. 10,1983,12 pp. Z Zbl 0515.14027 MR 0720556 